\title{
Refixation Patterns of Mind-wandering during Real-world Scene Perception
}

\author{
Han Zhang ${ }^{1}$, Nicola C. Anderson ${ }^{2}$, and \& Kevin F. Miller ${ }^{1}$ \\ ${ }^{1}$ University of Michigan \\ ${ }^{2}$ University of British Columbia
}

\begin{abstract}
Recent studies have shown that mind-wandering (MW) is associated with changes in eye movement parameters, but have not explored how MW affects the sequential pattern of eye movements involved in making sense of complex visual information. Eye movements naturally unfold over time and this process may reveal novel information about cognitive processing during MW. The current study used Recurrence Quantification Analysis (Anderson et al., 2013) to describe the pattern of refixations (fixations directed to previously-inspected regions) during MW. Participants completed a real-world scene encoding task and responded to thought probes assessing intentional and unintentional MW. Both types of MW were associated with worse memory of the scenes. Importantly, RQA showed that scanpaths during unintentional MW were more repetitive than during on-task episodes, as indicated by a higher recurrence rate and more stereotypical fixation sequences. This increased repetitiveness suggests an adaptive response to processing failures through re-examining previous locations. Moreover, this increased repetitiveness contributed to fixations focusing on a smaller spatial scale of the stimuli. Finally, we were also able to validate several traditional measures: both intentional and unintentional MW were associated with fewer and longer fixations; Eye-blinking increased numerically during both types of MW but the difference was only significant for unintentional MW. Overall, the results advanced our understanding of how visual processing is affected during MW by highlighting the sequential aspect of eye movements.

Public Significance Statement Recent research has found that MW is associated with changes in eye movements. We extended previous research by examining how eye movements unfold over time. In a scene perception task, scanpaths were more repetitive during unintentional MW compared to on-task episodes, suggesting an adaptive response to processing failures by re-examining previous locations. We also found that fixations during $\mathrm{MW}$ were fewer in number, longer in duration, and less spread in space. MW was also associated with increased eye-blinking. Overall, these results advance our understanding of the mechanisms of MW and demonstrate how scanpaths can play a role in developing tools to diagnose moments of inattention.
\end{abstract}

Keywords: mind-wandering; scanpath; refixations; scene perception; eye-tracking Word count: 8933

Mind-wandering (MW) is an attentional state in which individuals have thoughts unrelated to the current task. ${ }^{1}$ The successful completion of many daily tasks (e.g., reading, driving, etc.) requires us to pay sufficient attention; the absence of which may cause low performance or even fatal tragedies.

Han Zhang, Combined Program in Education and Psychology, University of Michigan. Nicola C. Anderson, Department of Psychology, University of British Columbia. Kevin F. Miller, School of Education and Department of Psychology, University of Michigan. Data, code, and stimuli are openly accessible at https://osf.io/6pj9m/.

Correspondence concerning this article should be addressed to Han Zhang, 610 E. University Avenue, Room 1413, Ann Arbor, MI, 48109. E-mail: hanzh@umich.edu
Unfortunately, MW appears to be a ubiquitous phenomenon across a wide range of cognitive tasks (Kane, Gross, et al., 2017; Kane et al., 2007; Lindquist \& McLean, 2011; Seli, Beaty, et al., 2018; Smallwood, McSpadden, et al., 2008; Yanko \& Spalek, 2014). A common way of measuring MW is using thought probes that occasionally appear during the

\footnotetext{
${ }^{1}$ It is worth noting that the definition of MW has recently become a hotly debated topic in the field (Christoff et al., 2018; Seli, Kane, Smallwood, et al., 2018; Seli, Kane, Metzinger, et al., 2018). Some have argued that in order to be qualified as MW, thoughts must proceed in a relatively free and unconstrained fashion (Christoff et al., 2018), whereas others disagree and instead argue that MW is a family of related mental phenomena that do not necessarily share a core set of features (Seli, Kane, Smallwood, et al., 2018; Seli, Kane, Metzinger, et al., 2018). The current study defines MW as thoughts unrelated to the current task at hand, without considering the level of constraints of these thoughts.
} 
task to inquire about participants' current focus. Using this technique, studies have shown that MW is associated with reduced performance in tasks that require sustained attention, including reading (Smallwood, 2011), problem-solving (Mrazek et al., 2012), attending to lectures (Kane, Smeekens, et al., 2017), driving (Yanko \& Spalek, 2014), and so on (for a review, see Mooneyham \& Schooler, 2013).

Why does task performance often suffer during MW? Previous research indicates that MW entails a decoupled state between attention and ongoing perceptual information (Schooler et al., 2011). For example, Kam et al. (2011) asked participants to complete a go/no-go task with a novel distractor inserted in a subset of the trials while their cortical responses were measured using electroencephalography. Self-reported attentional states were obtained using thought probes during the task. They found that participants had reduced cortical response to both the target and the sudden onset of the distractor (as measured by the P3 and P1 eventrelated potential component) during mind-wandering compared to when being on-task. Other studies have similarly found that MW was associated with an attenuation of perceptual processing, as measured by event-related potentials (Barron et al., 2011; Braboszcz \& Delorme, 2011; Smallwood, Beach, et al., 2008), task-evoked pupillary responses (Jubera-García et al., 2019; Unsworth \& Robison, 2016, 2016), and brain activation patterns (Fox et al., 2015). Perceptual decoupling has been suggested to play a functional role in protecting the continuity and integrity of the internal train of thought by minimizing perceptual interference (Smallwood, 2013). But when there is an important task at hand, it may lead to undesirable outcomes.

\section{Mind-wandering and Eye Movements}

Processing the visual world around us constitutes an important part of our daily lives. Gaze control has been extensively studied in the context of processing real-world scenes (Henderson, 2003; Land \& Hayhoe, 2001; Oliva \& Torralba, 2006; Rayner et al., 2009; Tatler et al., 2011; Torralba et al., 2006). Fixations naturally unfold over time and our understanding of a scene is not only built up from where and for how long we look, but also the particular sequential order in which we look at it. This sequence of eye movements has long been thought to reflect the cognitive processes occurring during scene viewing. Early work by Yarbus (1967) showed that scanpaths on the same painting unfolded differently depending on the task goal. Noton and Stark (1971a, 1971b)'s scanpath theory further proposed that scanpaths are an external reflection of the viewer's internal cognitive representation. Past research has shown that scanpath signatures can reflect differences in fixation strategies (Malsburg et al., 2015; Malsburg \& Vasishth, 2011), the nature of the task (Dewhurst et al., 2018; Foulsham \& Underwood, 2008), as well as in- trinsic abilities among viewers (Malsburg \& Vasishth, 2013; McIntyre \& Foulsham, 2018).

Interestingly, past research indicates that our eyes may continue to move even without the presence of visual stimuli (e.g., Altmann, 2004; Ehrlichman et al., 2007; Johansson \& Johansson, 2014; Spivey \& Geng, 2001). For example, eye movements are driven to previous locations even when the actual stimulus is removed (Altmann, 2004). This suggests that eye movements need not be driven only by the current visual processing needs. An often unspoken assumption of gaze control models is that the viewer is processing the scene without any influence from task-unrelated thoughts. This can be an optimistic assumption in either lab or daily life settings, as previous studies have shown (Kane, Gross, et al., 2017; Kane et al., 2007; Lindquist \& McLean, 2011; Seli, Beaty, et al., 2018; Smallwood, McSpadden, et al., 2008; Yanko \& Spalek, 2014). MW thoughts introduce a top-down interference to the attentional system, which then must seek to find a new balance between internal (task-irrelevant) processing versus perceptual (task-relevant) processing (Krasich et al., 2018). The consequence of this balance shift might be a breakdown of the "normal" moment-to-moment gaze control process, which may lead to observable changes in scanpath patterns.

Over the past few years, an increasing number of studies have investigated the relationship between eye movements and MW (e.g., Faber et al., 2020; Foulsham et al., 2013; Frank et al., 2015; Krasich et al., 2018; Reichle et al., 2010; Steindorf \& Rummel, 2020; Uzzaman \& Joordens, 2011; Zhang et al., 2020), but so far little attention has been given to scanpaths. Moreover, the majority of those studies were concerned with reading comprehension, which involves quite different gaze control mechanisms (Castelhano \& Rayner, 2008; Rayner et al., 2009). Therefore, it does not necessarily follow that MW would manifest in a similar set of eye movement patterns in scene perception. In fact, even within the reading domain, the results are not entirely consistent (discussed further in later sections).

To our knowledge, two previous studies examined MW in the context of scene perception. Krasich et al. (2018) presented participants with a series of real-world scenes to study and occasionally presented a thought probe in the middle of the presentation to inquire about participants' attentional state. They analyzed eye movements shortly before the probe presentation and found that MW was associated with fewer and longer fixations on the scene. These fixations also appeared to have a greater spatial spread (fixations located further away from each other) compared to on-task episodes. These results were successfully replicated in a subsequent experiment. A second study (Faber et al., 2020) used a similar paradigm but was only able to replicate the reduced number of fixations during MW. Overall, these results are encour- 
aging as they show that gaze control during scene perception undergoes observable changes during MW. But the inconsistencies between studies also call for further evaluation of the moment-to-moment gaze patterns during MW.

\section{Recurrence Quantification Analysis}

The current work focuses on one aspect of scanpaths: the pattern of refixations (i.e., fixations returning to previouslyviewed locations). Refixations are an important part of normal gaze behavior across different tasks and have been shown to play a functional role in remembering and comprehending information (e.g., Schotter et al., 2014; Meghanathan et al., 2019; Zelinsky et al., 2011). Recently, Anderson et al. (2013) introduced Recurrence Quantification Analysis (RQA) to capture the temporal pattern of refixations. RQA is a method to understand the temporal structure of a single scanpath (unlike other scanpath measures that require comparisons between two different sequences of eye movements). This feature makes RQA measures relatively easy to interpret and is convenient for statistical testing. The calculation of RQA measures is also independent of the specific content of the stimuli, making them potential candidates of task-general predictors of MW (Faber, Bixler, et al., 2018).

Two fixations are considered recurrent if their physical distance is below a pre-defined threshold (usually $1^{\circ}-2^{\circ}$ of visual angle corresponding to foveal vision; Anderson et al., 2013). For a given fixation sequence, we can illustrate all recurrent fixations at all time lags on a plot like the one in the panel $a$ of Figure 1. In this recurrence plot, the X- and Yaxis represent the same fixation sequence, with the numbers representing the fixation index (e.g., 1st fixation, 9th fixation, etc.). Recurrent fixations are denoted as a black dot. Each fixation is recurrent with itself, as shown by the major diagonal line. Above the line of self-recurrence, we can see multiple cases where later fixations are recurrent with earlier fixations (e.g., fixations 11, 19, and 20 are recurrent with fixation 1).

From the recurrence plot, Anderson et al. (2013) identified and described four recurrence measures which are briefly outlined here (also see Gurtner et al., 2019). First, the recurrence rate indicates the percentage of recurrent fixations for a given fixation sequence. It indicates how often a viewer returns to previously-inspected areas. Second, determinism indicates the percentage of recurrent points that fall on diagonals parallel to the line of self-recurrence (as illustrated in Figure 1, panel $b$ ). Determinism increases when a viewer moves the gaze from area $\mathrm{A}$ to $\mathrm{B}$ to $\mathrm{C}$ and later repeats the exact same sequence in the same order. Therefore, determinism represents repeated fixation sequences. Third, laminarity indicates the percentage of recurrent fixations that fall on horizontal or vertical lines in the recurrence plot (as illustrated in Figure 1, panel $c$ ). Vertical lines represent cases where an area is first examined by a single fixation and later on examined by consecutive fixations. In contrast, horizontal lines represent cases where an area is first examined with multiple fixations and later on re-examined with a single refixation. For determinism and laminarity, usually a minimum line-length threshold of 2 is set to rule out single refixations. Fourth, the center of recurrence mass (CORM) measures the overall position of recurrence points in relation to the line of self-recurrence. A small CORM value indicates that refixations occur relatively close in time (i.e., close to the line of self-recurrence). For example, if a viewer tends to immediately re-examine a previous location, the recurrent points will be close to the line of self-recurrence, producing a small CORM value. Alternatively, if a viewer tends to re-examine a previous location after many intermediate fixations at other locations, the recurrence points will be far from the line of self-recurrence, producing a large CORM value.

To summarize, the recurrence rate (overall percentage of refixations) and CORM (whether refixations occur soon or late) capture the global pattern of recurrences in a scanpath. Determinism (the percentage of repeated fixation sequences) and laminarity (the percentage of detailed inspections) further characterize the local pattern of recurrences.

How would the pattern of refixations change during MW? One possibility is that the viewer tends to skim over the entire picture without detailed processing at previous locations. This is in line with research demonstrating that repeated fixations are a normal part of ongoing language processing. When such processing is disrupted, by having observers view meaningless symbols rather than words, observers spend less time refixating previously-seen words, or those they had skipped (Luke \& Henderson, 2013; Rayner \& Fischer, 1996). If repeated fixations are a sign of normal, ongoing image encoding, we may expect to see a decline in such repetitions during MW as visual processing becomes superficial. Alternatively, one can predict that deficits in visual processing should make refixations more likely to occur to compensate for processing deficits. Refixations are known to be an adaptive response to insufficient processing of information at previous locations (Droll \& Hayhoe, 2007; Gilchrist \& Harvey, 2000; Körner \& Gilchrist, 2008; Meghanathan et al., 2019; Tatler et al., 2005; Zelinsky et al., 2011). For example, Tatler et al. (2005) showed that refixations can serve to update the retained description of a previously-seen object. Zelinsky et al. (2011) also proposed that refixations serve a rehearsal function to refresh fading object representations in visual working memory. Moreover, repeated scanpaths (instead of single re-fixations) have also been shown to aid the rehearsal (Tremblay et al., 2006) and retrieval (Bochynska \& Laeng, 2015) of visual-spatial information. In terms of RQA measures, recurrence and determinism became larger with increased working memory load (Meghanathan et al., 2019) 
a.

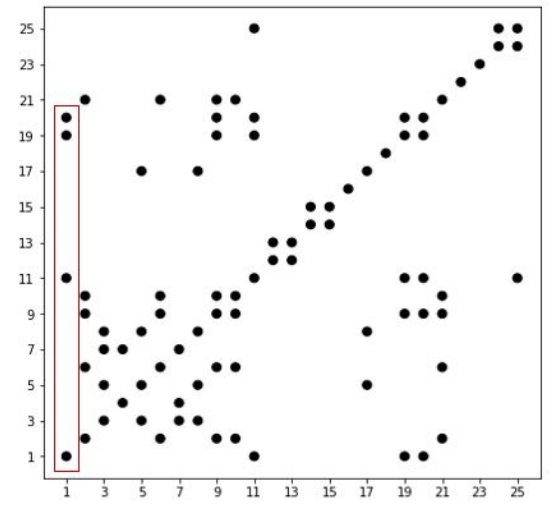

b.

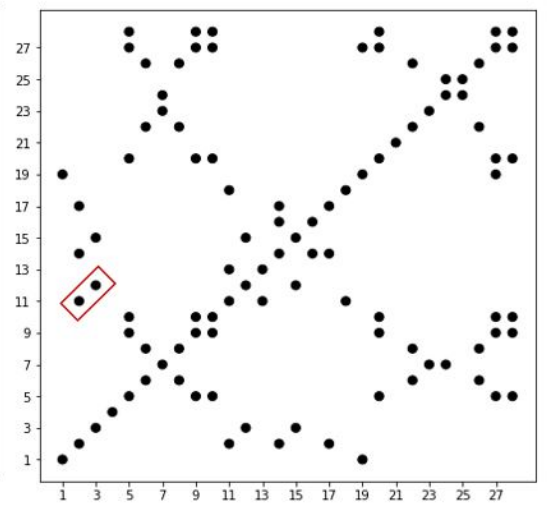

c.

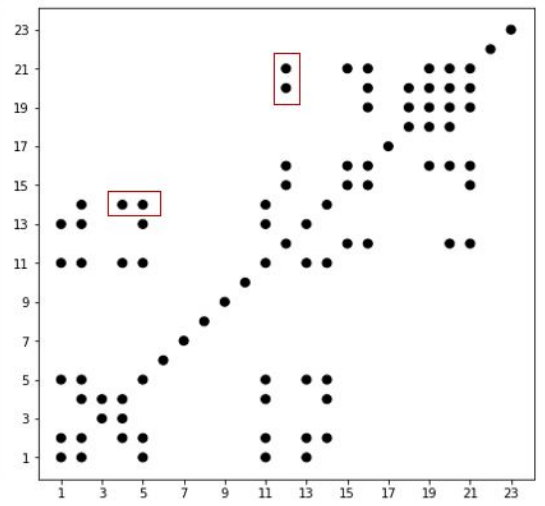

Figure 1

Example of recurrence plots. The $X$ - and Y-axis represent the same fixation sequence, with the numbers representing the fixation index (e.g., 1st fixation, 9th fixation, etc.). Two fixations are considered recurrent (denoted as a black dot) if their physical distance is below a pre-defined threshold (usually $1^{\circ}-2^{\circ}$ of visual angle corresponding to the foveal vision; Anderson et al., 2013). The red rectangle in Panel a illustrates that fixations 11, 19, and 20 are recurrent with fixation 1. Panel b illustrates determinism. As highlighted in the red rectangle, areas examined by fixations 2 and 3 are re-examined later in this trial in the exact same order by fixations 11 and 12. Panel c illustrates laminarity. As highlighted in the red rectangles, an area examined by fixation 12 is later re-examined by fixations 20 and 21, forming a vertical line. In contrast, an area initially examined by fixations 4 and 5 is later re-examined by fixation 14, forming a horizontal line. Finally, compared to panel b, the recurrent fixations in panel $\mathrm{c}$ are closer to the major diagonal, meaning that refixations in panel coccur closer together in the fixation sequence. Therefore, the center of recurrence mass (CORM) is smaller in panel c than in panel b.

and during mental imagery (imagined scene must be reactivated repeatedly in the absence of the actual display; Gurtner et al., 2019). In sum, previous literature also points to increased scanpath repetitiveness during MW. These two possibilities are in opposite directions, but both seem to be sensible eye movement patterns in response to the reduced visual processing during MW. Notably, aggregated eye movement measures do not permit distinguishing between these two predictions, but scanpath analysis can reveal how MW affects the way that looking unfolds over time.

\section{Aggregated Measures}

Although the present work is primarily concerned with scanpaths, we also examined several aggregated measures that have been reported to be associated with MW. In particular, we are interested in the spatial spread of fixations. A high fixation dispersion indicates that fixations are located far away from each other, whereas a low dispersion indicates that fixations are constrained to certain areas of the scene. Previous studies reported inconsistent results on the relationship between fixation dispersion and MW. Krasich et al. (2018) reported a marginally significant increase in fixation dispersion during MW in a scene perception task, and this effect was significant in a subsequent replication. The effect was not significant in another study that also used a scene perception task (Faber et al., 2020). In a virtual tutor setting, Hutt et al. (2017) reported greater fixation dispersion during MW. But Zhang et al. (2020) found just the opposite in a video lecture setting, although this analysis was only constrained to the main area where the slides were displayed. These inconsistent results muddy the interpretation of this measure. We argue that to make sense of fixation dispersion, it is vital to examine the recurrence pattern of fixations. Recall that recurrence is defined based on the physical distance between two fixations. Therefore, a repetitive scanpath would imply that some fixations are very close to each other, which may contribute to a lower spatial spread. The current study sought to answer both if MW is associated with the spatial spread of fixations and how the eventual pattern is generated step-bystep. To the extent that scanpaths are more repetitive during $\mathrm{MW}$, the overall spatial spread of fixations should be smaller.

We also examined the number of fixations and the mean duration of fixations. Having fewer fixations indicates an overall withdrawal of visual sampling, which is what the perceptual decoupling theory would predict in tasks requiring external attention (e.g., reading and scene perception). Interestingly, previous studies have not been entirely consistent on this measure. Some studies reported that MW was associated with fewer fixations during reading and scene perception (Faber et al., 2020; Krasich et al., 2018), whereas other reported no difference (Uzzaman \& Joordens, 2011), or even 
more fixations during MW (Foulsham et al., 2013; Steindorf \& Rummel, 2020). A similar mixture of findings has been reported for fixation duration. Some studies found longer fixation duration during MW in reading or in scene perception (Foulsham et al., 2013; Krasich et al., 2018; Smilek et al., 2010), whereas others did not (Faber et al., 2020; Uzzaman $\&$ Joordens, 2011). Fixation duration and count are usually negatively correlated given a fixed period, because spending longer time at each location reduces the chance of having more fixations. From a theoretical standpoint, increased fixation duration may indicate that the viewer needs longer time to analyze incoming information before moving to the next location (Tatler et al., 2017). Such cases may be more likely to occur during MW due to deficiencies in perceptual processing.

Finally, we examined the number of blinks during MW. Smilek et al. (2010) reported that readers blinked more often when they were mind-wandering than when they were ontask during reading comprehension. The authors suggested that blinking might serve to protect the integrity of internal thoughts by blocking perceptual input for a brief moment. The theory is attractive in that it interprets blinking as an overt embodiment of the perceptual decoupling process. However, subsequent studies obtained mixed results (Faber, Bixler, et al., 2018; Grandchamp et al., 2014; Krasich et al., 2018; Uzzaman \& Joordens, 2011). Importantly, other types of tracking loss, such as a substantial change in the head position and micro-sleeps, can be confounded with true blinks. If these abnormal tracking episodes are more likely to happen during MW (and there are reasons to believe so; Caffier et al., 2003; Häkkänen et al., 1999; Schleicher et al., 2008; Seli et al., 2014), the true effect size associated with blinking might be inflated. In this study, we detected eyeblinking using a novel algorithm developed by Hershman et al. (2018). The algorithm detects blinks based on the change in pupil size between subsequent samples and was shown to be more accurate than some of the currently-used methods. We examined both blinks in a range considered to be typical (50 - $500 \mathrm{~ms}$; Wang et al., 2011), and those beyond the range. This practice may lead to a better estimation of the true effect size associated with blinking.

\section{Intentional vs. Unintentional MW}

Recent studies have shown that MW can occur with or without intention (for a review, see Seli, Risko, Smilek, et al., 2016). Unintentional MW occurs when individuals lose control over their thoughts despite their best intention to focus on the task, whereas intentional MW occurs when individuals decide to disengage from the task. While both intentional and unintentional MW entail task-unrelated thoughts, studies have shown that they are associated with different psychological and neurological processes. For example, increasing task difficulty reduces the rate of intentional MW but increases the rate of unintentional MW (Seli, Risko, \& Smilek, 2016); task motivation and interest appear to be more strongly associated with intentional MW than with unintentional MW (Phillips et al., 2016; Seli, Cheyne, et al., 2015; although see Seli et al., 2017). People with ADHD, a group characterized by heightened distractibility, were found to experience more unintentional MW but not more intentional MW (Seli, Smallwood, et al., 2015). Trait-level intentional and unintentional MW were also found to have distinct neural correlates (Golchert et al., 2017). These findings motivated us to examine whether intentional and unintentional MW are dissociable in gaze parameters.

How might intentional and unintentional MW differ in gaze parameters? We note that previous research does not offer clear suggestions, and we can only make some speculations. By definition, intentional MW is a more deliberate and controlled process compared to unintentional MW. Individuals during intentional MW may be fully aware that they are offtask but still decide to stay in this mode. The deliberate detachment from the task-goal means that individuals may not take necessary steps, such as refixating a previous location, to repair visual processing. But in the case of unintentional MW, individuals unwillingly drift off from the task despite their best intention to stay on-task. The presence of some latent task goal may therefore prompt efforts to repair failed visual processing in the form of refixations. Therefore, we speculate that the differences between intentional and unintentional MW may be captured by the recurrence measures. In particular, intentional and unintentional MW may differ in the overall recurrence rate as well as the local repetition patterns (e.g., lower determinism and/or lower laminarity).

\section{The Current Study}

In the present work, we asked participants to study a range of real-world scenes for a later memory test. Thought probes were occasionally presented to inquire about participants' attentional state. We analyzed gaze parameters associated with the pictures presented just before the thought probes. Specifically, we examined four novel measures capturing the sequential aspect of fixations: recurrence rate, determinism, laminarity, and CORM. We also re-examined several aggregated measures previously reported to be associated with MW, including fixation dispersion, fixation count, fixation duration, and the number of blinks. For each measure, we examined how it might change when a viewer is on-task compared to when they are intentionally or unintentionally mindwandering. A final set of comparisons examined potential differences between intentional and unintentional MW. 


\section{Method}

\section{Sample Size Planning}

The statistical power of the current design is related to the number of participants, the number of probes, as well as the actual MW rate. Ideally, a study should strive for a large number of participants with a large number of thought probes, while ensuring that the probes are not so intrusive as to inhibit MW (Seli et al., 2013; although see Robison et al., 2019). Considering the constraints in time, lab space, and the research assistants available, we decided that collecting a large number of participants was not feasible for us. Therefore, we decided to increase the number of thought probes per participant, while trying to collect as many participants as we could. We set our goal to collect 60 participants with 36 thought probes per participant. Thought probes were designed to be separated by at least 30 seconds, as we consider this as a reasonable interval based on previous research (Seli et al., 2013). Figure A1 in the Appendix shows where we are relative to past studies in terms of the number of participants and the number of probes: The number of participants was around the median level but the number of probes was larger compared to the majority of studies.

\section{Participants}

We recruited 64 undergraduate students (Age: $M e a n=18.84$, $S D=.79 ; 64 \%$ female; all with normal eyesight) from the University of Michigan to participate for course credit. After discarding data from 7 participants who had low tracking ratios ( $<75 \%$, a threshold set a priori), the final sample size is 57 participants.

\section{Apparatus and Stimuli}

\section{Pictures}

We selected 180 pictures as the to-be-studied material from the SUN database (Xiao et al., 2010) and the LabelMe database (Russell et al., 2008). The stimuli set consisted of 60 exteriors, 60 interiors, and 60 landscapes. We selected only pictures without identifiable human faces. Of these pictures, we randomly selected 12 of each type (36 total) as the probed pictures, meaning that they will be followed by a thought probe (discussed below). The probed pictures were the same across participants. Another 72 pictures from the same databases (24 for each type) were selected as new pictures (lures) in the memory test. All pictures were presented in $1024 * 768$ pixels on a 20.1 -inch screen at approximately $70 \mathrm{~cm}$ to the participant. Thus, each picture subtended about $32^{\circ} * 25^{\circ}$ of visual angle.

\section{Thought Probes}

A thought probe was presented after every target picture, asking "where was your attention during the last picture?".
Participants chose either "I was focusing on the picture" by pressing "A" on the keyboard or "I was thinking about something else" by pressing " $\mathrm{B}$ " on the keyboard. If the latter was chosen, participants were further asked to indicate their intentionality of MW by pressing "A" for intentional MW and "B" for unintentional MW. "On-task" was communicated to the participants as "you were focused on completing the task and were not thinking about anything unrelated to the task"; MW was communicated to the participants as "you were thinking about something completely unrelated to the task", with intentional MW as "you intentionally decided to think about things that are unrelated to the task" and unintentional MW as "your thoughts drifted away despite your best intentions to focus on the task" (Seli, Cheyne, et al., 2015).

\section{Eye-tracking}

Monocular eye movements were recorded by an Eyelink 1000 tracker at a sampling rate of $500 \mathrm{~Hz}$. No chin rest was used and head movement was adjusted by tracking a sticker on the participant's forehead. The experiment was implemented by the OpenSesame software (Mathôt et al., 2012) with functions from the PyGaze package (Dalmaijer et al., 2014). Saccades were detected based on a velocity threshold of $30 \hat{\mathrm{A}} \% \mathrm{~s}$ and an acceleration threshold of $8000 \hat{\mathrm{A}} \% \mathrm{~s} / \mathrm{s}$.

\section{Procedure}

After signing the informed consent form, participants were asked to study pictures for a memory test. Participants learned that a thought probe would occasionally occur after some of the pictures and ask them whether they were mindwandering or on-task during their viewing of the picture just presented. Participants first completed a practice block that consisted of 5 example trials (with a thought probe) and a recognition test. Then, the experimental blocks began. Exteriors, interiors, and landscapes were presented in three separate blocks. The block order was counterbalanced across participants. Each block had a study-test structure. In the study phase of each block, participants studied 60 pictures consecutively. Each picture was presented for 10 seconds. The structure of each trial is shown in Figure 2. A thought probe was presented after every probed picture. The order of presentation was randomized with the constraint that any two probed pictures were separated by at least three non-probed pictures. In the test phase of each block, participants saw a series of pictures and indicated whether each picture was an old one or a new one. The test set consisted of all 12 probed pictures, 12 randomly-selected non-probed pictures from the current block, and 24 pictures that were not presented but belongs to the same type (lures). After the test phase, participants took a short break before starting the next block. The eye-tracker was calibrated using a 5-point calibration before each block. 


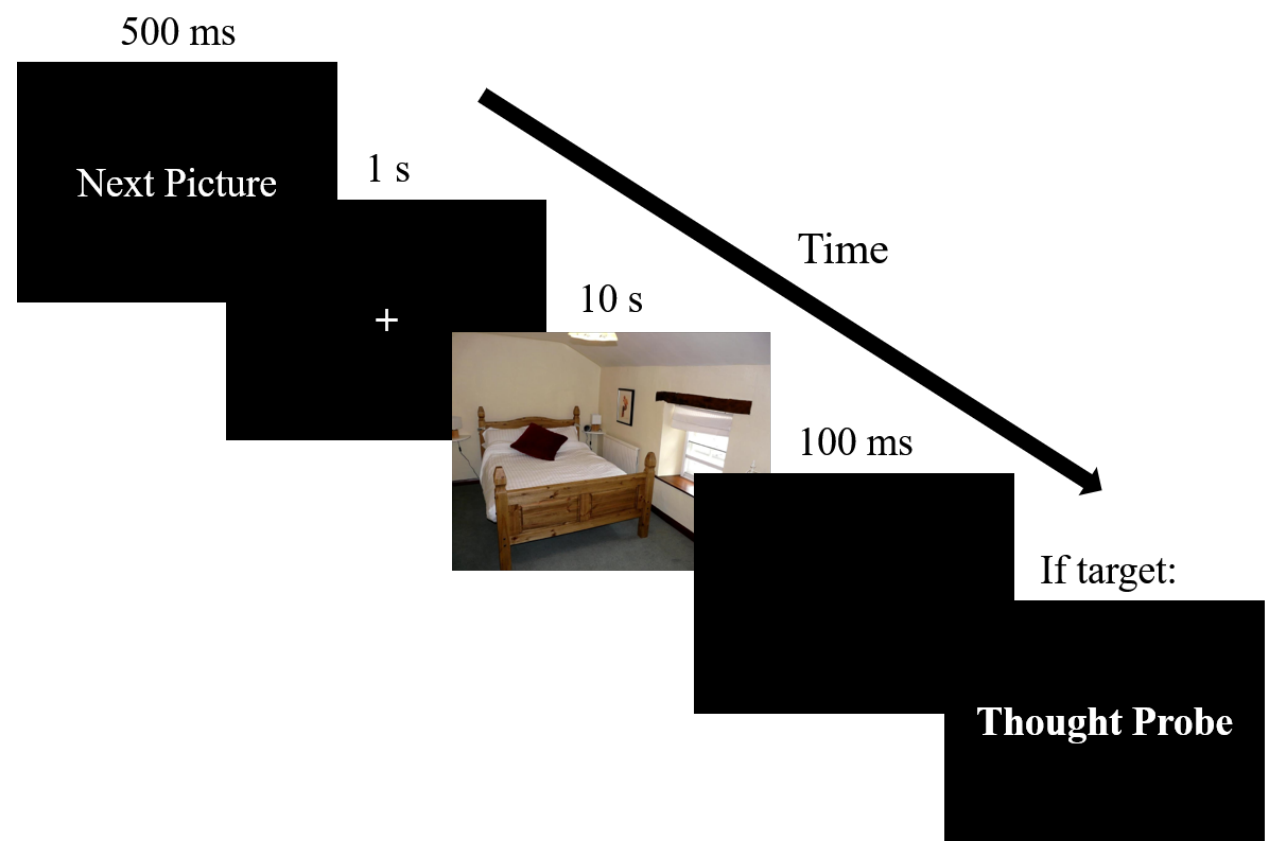

\section{Figure 2}

An example trial during the study phase. A trial started with a 500 milliseconds display of "Next Picture", followed by a 1-second fixation cross. Then the picture appeared for 10 seconds, followed by a black screen for 100 milliseconds. For target trials, a thought probe would then appear to ask whether participants were mind-wandering during the picture they just saw.

\section{Data Analysis}

Data analysis was conducted in $\mathrm{R}$ (Version 3.6.1; R Core Team, 2019) $)^{2}$. Although we tracked eye movements during all stimuli, we only analyzed the probed trials. All probe trials were included irrespective of the correctness of the response in the test phase. We discarded fixations greater than $2000 \mathrm{msec}$ or shorter than $80 \mathrm{msec}(8.1 \%$ of data) and fixations outside of the screen ( $1.82 \%$ of data) before computing eye movement measures. Trials with no fixations $(1.82 \%$ of trials) were also discarded. Recurrence measures were computed based on a radius of 60 pixels (about $2^{\circ}$ of visual angle; Anderson et al., 2013). A threshold of 2 consecutive recurrences (as default) was set to rule out single refixations in the calculation of determinism and laminarity. The code for computing RQA measures can be found at https://barlab.psych.ubc.ca/research/. Note that for determinism, laminarity, and CORM, zeroes were treated as missing per the program's default.

Because RQA measures are high-level, arbitrary values that are derived from fixation information, they require validation against random fixation sequences. This validation is especially important for the on-task condition, as we do not expect participants to browse the scene randomly if they reported being on-task. On the other hand, participants may browse the scene in a more random fashion during $\mathrm{MW}$, in which case the RQA measures may not be above chance level. For each attentional state, we computed fixation density maps using a two-dimensional Gaussian filter (window size $=150 * 150$ pixels, $S D=30$ pixels). We then created random fixation sequences by drawing samples from these fixation density maps. This was repeated for 1000 times for each trial. Then, for each attentional state, we compared the empirical RQA values against those calculated from these random fixation sequences. This would produce a distribution of $t$-values for each RQA measure under each attentional state. We declare an empirical value is above-chance if more than $95 \%$ of the $t$-values were above the critical value (critical $t=1.67$ when $\mathrm{N}=57$, one-tailed). Detailed results of this validation process can be found in Appendix A2. In short, all empirical values were above-chance except for determinism during intentional MW. Thus, there is some peculiarity in the

\footnotetext{
${ }^{2}$ We, furthermore, used the R-packages afex (Version 0.27.2; Singmann et al., 2019), corx (Version 1.0.6; Conigrave, 2020), emmeans (Version 1.4.8; Lenth, 2019), kableExtra (Version 1.1.0; Zhu, 2019), knitr (Version 1.29; Xie, 2015), lme4 (Version 1.1.23; Bates et al., 2015), lmerTest (Version 3.1.2; Kuznetsova et al., 2017), papaja (Version 0.1.0.9997; Aust \& Barth, 2020), qwraps 2 (Version 0.4.2; DeWitt, 2019), and tidyverse (Version 1.3.0; Wickham et al., 2019).
} 
way participants browsed the scene during intentional MW that prevented repetitions in scanpath sequences.

Responses to the thought probes were heavily unbalanced: participants on average reported being on-task for about $73 \%$ of the time $(S D=22 \%)$, followed by unintentional MW $($ Mean $=22 \%, S D=19 \%)$, and intentional MW (Mean $=$ $5 \%, S D=8 \%)$. The rate of unintentional MW did not significantly differ across picture types (exteriors vs. interiors: $b=$ -.009, $S E=.02, t=-.42, p=.67$; exteriors vs. landscapes: $b=$ -.04, $S E=.02, t=-1.80, p=.22$; interiors vs. landscapes: $b=$ $-.03, S E=.02, t=-1.37, p=.35$ ). The rate of intentional MW also did not differ across picture types (exteriors vs. interiors: $b=-.002, S E=.01, t=-.19, p=.85$; exteriors vs. landscapes: $b=-.02, S E=.01, t=-1.62, p=.33$; interiors vs. landscapes: $b=-.02, S E=.01, t=-1.42, p=.33)$. The overall MW rate is consistent with previous literature (Mean $=27 \%, S D=22 \%$ in Krasich et al., 2018), but the rate of intentional MW was unexpectedly low (Seli, Risko, \& Smilek, 2016; Seli, Carriere, et al., 2018; Seli, Cheyne, et al., 2015). Therefore, we suggest the reader be cautious when interpreting results regarding intentional MW due to limited observations.

We used linear mixed-effects models (Bates et al., 2015) to examine potential differences in gaze parameters between attentional states. Attention (intentional MW/unintentional MW/on-task) was dummy-coded with "on-task" being the reference level. Picture type (exteriors/interiors/landscapes) entered the model as a covariate. ${ }^{3}$ We started by fitting these linear mixed models with all possible random intercepts and slopes (Barr et al., 2013). In the case of non-convergence or singular fit, we performed backward elimination of random effects using the step () function from the lmerTest package (Kuznetsova et al., 2017) ${ }^{4}$. In the main text, we report pairwise comparisons of marginal means across attentional states $^{5} . p$-values were estimated using the Satterthwaite approximation and were further corrected for pairwise comparisons using the Holm-Bonferroni method. Detailed model summaries are included in the supplemental material.

\section{Results}

\section{Descriptive Statistics and Correlations of Dependent Variables}

Table 1 shows descriptive statistics (number of trials, means, and standard deviations) of dependent variables. Table 2 shows a correlation matrix of these variables.

\section{Recognition of Probed Pictures}

If participants were unintentionally mind-wandering rather than on-task during the presentation of pictures, they were less likely to recognize them during the memory test, $b=$ $-0.16, S E=0.02, t=-7.94, p<.001$. Similarly, participants had worse recognition if they were intentionally mind- wandering during the presentation of probed pictures, $b=$ $-0.16, S E=0.04, t=-4.54, p<.001$. There was no significant difference between intentional and unintentional $\mathrm{MW}, b$ $=-0.006, S E=0.04, t=-0.16, p=0.88$. The estimated hit rate for pictures associated with being on-task, unintentional $\mathrm{MW}$, and intentional MW was $.89(S E=.02), .74(S E=.02)$, and $.73(S E=.04)$, respectively.

\section{Gaze Parameters during Mind-wandering}

Figure 3 shows the estimated marginal means of RQA (panels $a$ to $d$ ) and aggregated (panels $e$ to $i$ ) measures under each attentional state, with significant differences marked by asterisks. In the sections below, we report the results for each measure in detail.

\section{Recurrence Rate}

The recurrence rate indicates the proportion of fixations directed to previously inspected areas. We found that recurrence rate was significantly higher during unintentional MW than during on-task episodes, $b=1.79, S E=0.64, t=2.80$, $p=0.02$. During intentional MW, recurrence rate was also numerically higher compared to on-task episodes but this difference was not significant, $b=3.50, S E=2.03, t=1.72, p$ $=0.19$. Intentional MW also did not significantly differ from unintentional MW on recurrence rate, $b=1.71, S E=2.10, t$ $=0.81, p=0.42$.

\section{Determinism}

Determinism indicates repeated fixation sequences. Unintentional MW was associated with a significantly higher determinism compared to on-task episodes, $b=4.03, S E=1.13$, $t=3.55, p<.001$. However, a similar pattern was not found for intentional MW, $b=-2.10, S E=2.04, t=-1.03, p=0.30$. Furthermore, determinism during intentional MW was significantly lower compared to during unintentional MW, $b=$ $-6.13, S E=2.18, t=-2.81, p=0.01$. Validation results (see Appendix A2) indicate that the level of determinism during intentional MW was as good as random.

\section{Laminarity}

Laminarity represents areas initially examined with a single fixation and later on followed-up with consecutive fixations or vice versa. There was no significant difference in laminarity between unintentional MW and on-task episodes, $b=$

\footnotetext{
${ }^{3}$ It might be interesting to examine the interaction between stimuli type and thought probe responses but this was complicated by the heavily unbalanced data.

${ }^{4}$ Two models still did not converge even after backward elimination, so we simply used a random intercept model with subject and picture identity.

${ }^{5}$ Results for unintentional MW would still hold when excluding intentional MW from analysis.
} 
Table 1

Descriptive Statistics ( $N$ of trials; Mean +/- SD) of Dependent Variables.

\begin{tabular}{llll}
\hline & On-task & Intentional MW & Unintentional MW \\
\hline Hit Rate $(0-1)$ & 1,$463 ; 0.90 \pm 0.30$ & $100 ; 0.70 \pm 0.46$ & $428 ; 0.71 \pm 0.45$ \\
Recurrence Rate $(\%)$ & 1,$459 ; 10.12 \pm 7.49$ & $100 ; 13.27 \pm 14.05$ & $424 ; 12.43 \pm 11.05$ \\
Determinism & 1,$265 ; 33.86 \pm 17.45$ & $82 ; 32.84 \pm 17.36$ & $331 ; 38.41 \pm 20.11$ \\
Laminarity & 1,$364 ; 26.77 \pm 15.74$ & $84 ; 29.08 \pm 16.97$ & $379 ; 27.19 \pm 16.11$ \\
CORM & 1,$447 ; 30.75 \pm 9.32$ & $96 ; 32.06 \pm 11.05$ & $418 ; 31.33 \pm 10.91$ \\
Fixation Dispersion & 1,$459 ; 0.36 \pm 0.09$ & $100 ; 0.32 \pm 0.10$ & $424 ; 0.33 \pm 0.10$ \\
Area Covered (0-1) & 1,$463 ; 0.22 \pm 0.07$ & $100 ; 0.18 \pm 0.07$ & $428 ; 0.18 \pm 0.07$ \\
N of Fixations & 1,$463 ; 24.60 \pm 7.33$ & $100 ; 20.18 \pm 8.01$ & $428 ; 19.89 \pm 7.67$ \\
Mean Fixation Duration & 1,$463 ; 313.93 \pm 99.89$ & $100 ; 355.10 \pm 178.37$ & $428 ; 366.00 \pm 161.70$ \\
N of Blinks & 1,$463 ; 2.68 \pm 2.22$ & $100 ; 3.72 \pm 2.45$ & $428 ; 3.16 \pm 2.45$ \\
\hline
\end{tabular}

Note. Results were based on trial-level data.

Table 2

Correlation Table for Dependent Variables

\begin{tabular}{|c|c|c|c|c|c|c|c|c|c|}
\hline & 1 & 2 & 3 & 4 & 5 & 6 & 7 & 8 & 9 \\
\hline 1. Hit Rate & - & & & & & & & & \\
\hline 2. Fixation Count & $.38 * *$ & - & & & & & & & \\
\hline 3. Mean Fixation Duration & $-.33^{*}$ & $-.82 * * *$ & - & & & & & & \\
\hline 4. Fixation Dispersion & $.40 * *$ & $.54 * * *$ & $-.34 * *$ & - & & & & & \\
\hline 5. Area Covered & $.46^{* * *}$ & $.89 * * *$ & $-.66^{* * * *}$ & $.79 * * *$ & - & & & & \\
\hline 6. Blinks & -.21 & -.04 & -.07 & -.25 & -.17 & - & & & \\
\hline 7. Recurrence Rate & $-.45 * * *$ & $-.53 * * *$ & $.36 * *$ & $-.79 * * *$ & $-.79 * * *$ & $.39 * *$ & - & & \\
\hline 8. Determinism & -.25 & $-.53 * * *$ & $.48 * * *$ & $-.48 * * *$ & $-.67 * * *$ & $.29 *$ & $.67 * * *$ & - & \\
\hline 9. Laminarity & -.11 & -.13 & .01 & $-.47 * * *$ & $-.45 * * *$ & $.34 *$ & $.71 * * *$ & $.71 * * *$ & - \\
\hline 10. CORM & $-.36 * *$ & -.22 & .21 & $-.35 * *$ & -.24 & .02 & .16 & $-.30 *$ & $-.33 *$ \\
\hline
\end{tabular}

Note. The table shows subject-level correlations aggregated across stimuli $(\mathrm{N}=67)$. $* \mathrm{p}<0.05 ; * * \mathrm{p}<0.01$; $* * * \mathrm{p}<0.001$

1.04, $S E=0.93, t=1.12, p=0.72$, or between intentional MW and on-task episodes, $b=2.02, S E=1.72, t=1.17, p=$ 0.72 . Intentional and unintentional $\mathrm{MW}$ did not significantly differ from each other, $b=0.99, S E=1.83, t=0.54, p=0.72$.

\section{Center of Recurrence Mass (CORM)}

The center of recurrence mass (CORM) indicates whether refixations occur close in time. We did not find a significant difference between on-task and unintentional MW episodes, $b=-0.07, S E=0.56, t=-0.12, p=0.90$. There was also no significant difference between being on-task and intentional MW: $b=-1.37, S E=1.02, t=-1.34, p=0.54$. Finally, there was no significant difference between intentional and unintentional MW: $b=1.30, S E=1.08, t=1.20, p=0.54$.

To further illustrate the scanpath patterns, we plotted representative scanpaths during unintentional MW and on-task episodes in Figure 4. In this plot, each red point represents a single fixation. Recurrent fixations are connected by a black line. As shown in the figure, the scanpath during unintentional MW (on the right) was more repetitive compared to the on-task scanpath (on the left).

\section{Fixation Dispersion}

Fixation dispersion was computed as the root mean square of the Euclidean distance from each fixation to the average position of all fixations (Krasich et al., 2018). It is reported on a $0-1$ scale by normalizing on the maximum dispersion possible, with higher values indicating greater dispersion.

Fixation dispersion was significantly smaller during unintentional MW than during on-task episodes, $b=-0.02, S E=$ $0.005, t=-3.86, p<.001$. For intentional MW, fixation dispersion was also smaller compared to on-task episodes, $b=$ $-0.02, S E=0.01, t=-2.95, p=.006$. But there was no significant difference between intentional and unintentional MW, $b=-0.002, S E=0.01, t=-0.20, p=0.84$. 
a. Recurrence Rate (\%)

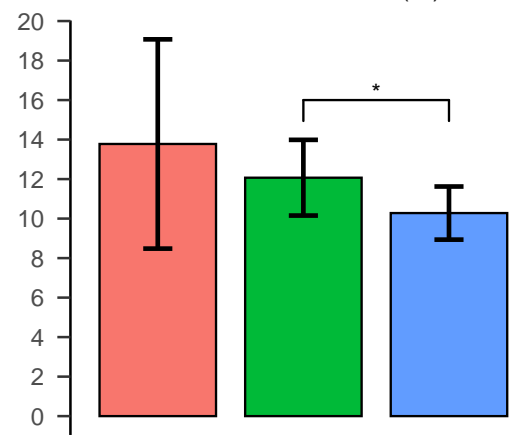

d. Center of Recurrence Mass (CORM)

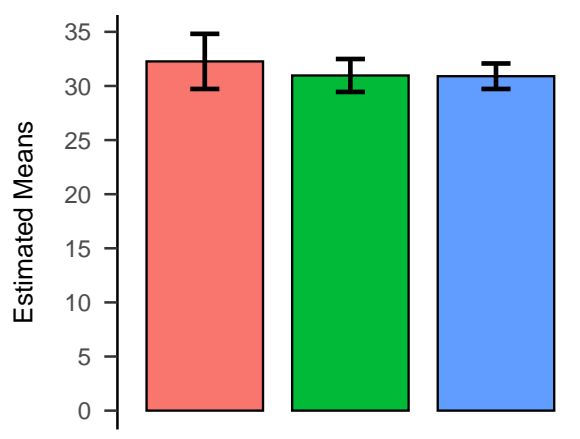

g. Number of Fixations

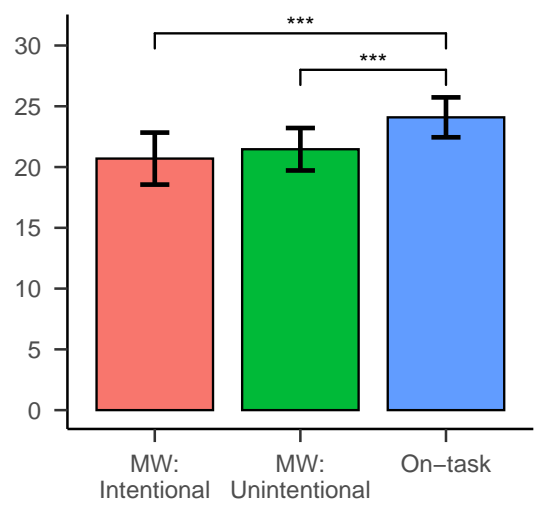

$\square \begin{aligned} & \text { MW: } \\ & \text { Intentional } \\ & \text { Unintentional } \\ & \text { Un: }\end{aligned}$ On-task

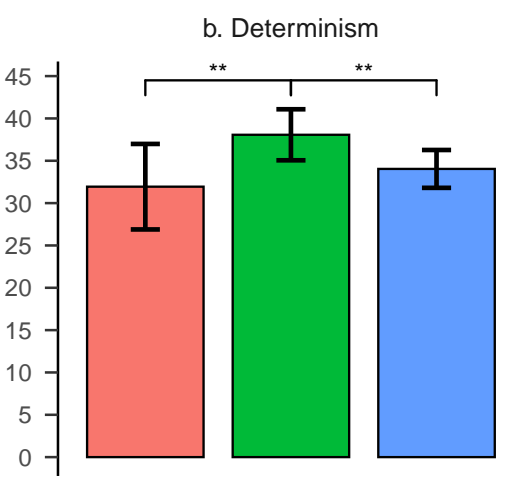

e. Fixation Dispersion

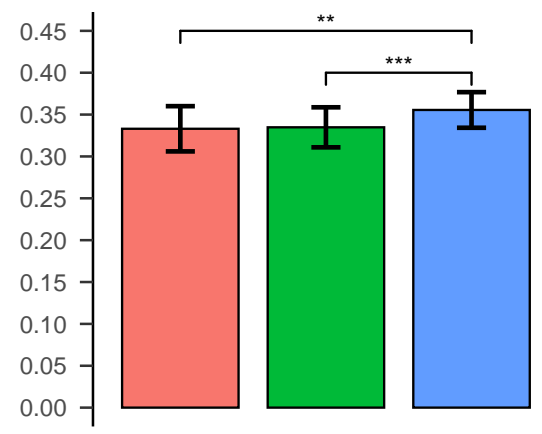

h. Mean Fixation Duration (msec)

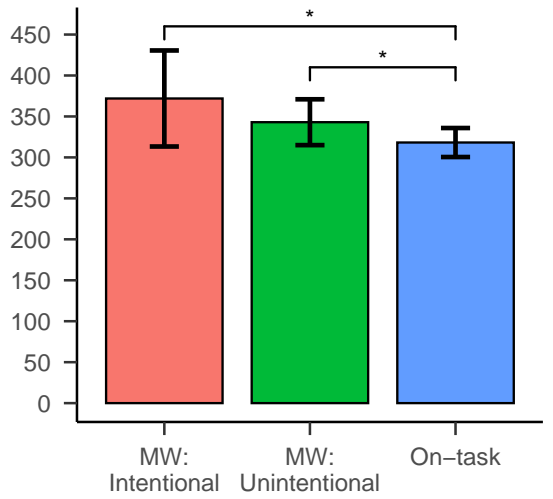

c. Laminarity

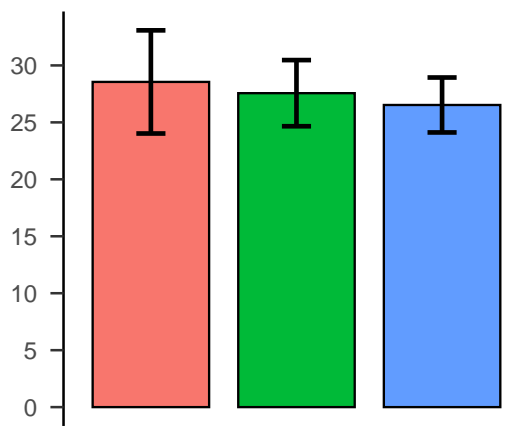

f. Covered Area

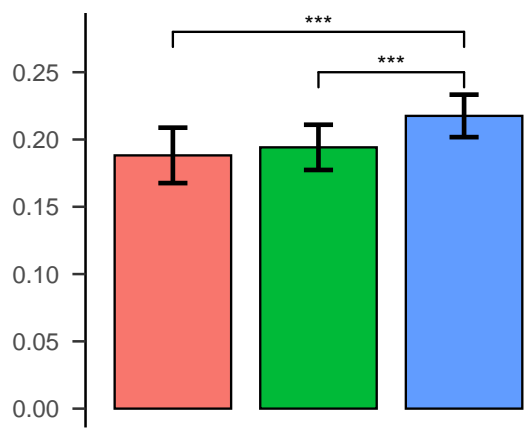

i. Number of Blinks

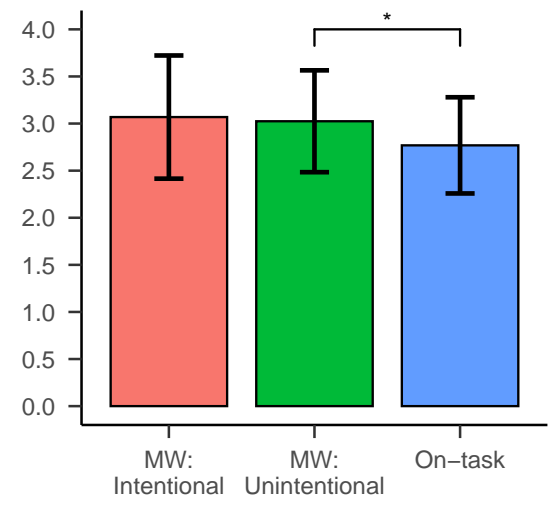

\section{Figure 3}

The estimated marginal means of eye-tracking measures during intentional MW, unintentional MW, and on-task episodes. Panels a to d show measures from Recurrence Quantification Analysis. Panels e and f show measures indicating the spatial property of fixations. Panel $\mathrm{g}$ to i show additional aggregated measures examined. Error bars show $95 \%$ confidence intervals. The annotations indicate the significance level of the difference between intentional/unintentional MW and the on-task episode. $* * p<.001, * * p<.01, * p<.05$.

\section{Area covered}

We computed another measure to indicate the spatial property of fixations. Specifically, we "drew" a circle with a ra- dius of 60 pixels (about $2^{\circ}$ of visual angle which is roughly the size of foveal vision) around each fixation and calculated the total area covered by these regions in proportion to the 
full picture size. If two fixation circles overlapped, we calculated the union of the area (without counting the overlapping regions repeatedly). Therefore, the possible range of this measure was between $0 \%$ to $100 \%$.

Unintentional MW was associated with less coverage compared to on-task episodes, $b=-0.02, S E=.003, t=-6.96, p<$ .001. A similar relationship was found for intentional MW, $b=-0.03, S E=0.01, t=-4.86, p<.001$. But intentional and unintentional MW did not significantly differ from each other, $b=-0.01, S E=0.01, t=-0.94, p=0.35$.

\section{Fixation Count}

Unintentional MW was associated with fewer fixations compared to on-task episodes, $b=-2.62, S E=0.35, t=-7.51, p$ $<.001$. A similar relationship emerged for intentional MW, $b=-3.39, S E=0.63, t=-5.40, p<.001$. There was no significant difference between intentional and unintentional $\mathrm{MW}, b=-0.77, S E=0.66, t=-1.16, p=0.25$.

\section{Fixation Duration}

The mean duration of fixations was longer during unintentional MW than during on-task episodes, $b=24.81, S E=$ 9.76, $t=2.54, p=0.04$. Similarly, the mean duration was longer during intentional $\mathrm{MW}$ than during on-task episodes, $b=53.75, S E=22.50, t=2.39, p=0.05$. We did not find a significant difference between intentional and unintentional $\mathrm{MW}, b=28.95, S E=24.10, t=1.20, p=0.24$.

\section{Eye-blinking}

We defined "normal" blinks to be those between $50 \mathrm{~ms}$ and 500 ms (Wang et al., 2011) and removed cases outside this range. This excluded $1.34 \%$ of all cases that were below 50 $\mathrm{ms}$ and $18.86 \%$ of all cases above $500 \mathrm{~ms}$. We found that unintentional MW was associated with more blinks compared to on-task episodes, $b=0.26, S E=0.10, t=2.45, p=0.04$. Intentional MW had numerically more blinks than on-task episodes but the difference was not statistically significant, $b$ $=0.30, S E=0.19, t=1.60, p=0.22$. Again, we did not find a significant difference between intentional and unintentional $\mathrm{MW}, b=0.04, S E=0.20, t=0.22, p=0.82$.

We further examined whether the number of overly long cases differed across attentional states. Unintentional MW was associated with more cases greater than $500 \mathrm{~ms}$ compared to on-task episodes, $b=0.18, S E=0.05, t=3.43, p=$ .002 . There was a numerically similar pattern for intentional MW compared to on-task episodes but the difference was not statistically significant, $b=0.17, S E=0.14, t=1.24, p=$ 0.44 . There was no significant difference between intentional and unintentional MW, $b=-0.01, S E=0.14, t=-0.06, p=$ 0.82 .

\section{Can Gaze Parameters Predict Memory?}

Our results so far suggest a relationship between gaze features when studying a picture and memory of that picture when it was later tested. As a secondary analysis, we examined how gaze features predicted subsequent memory and whether the relationship was moderated by MW. For each gaze feature, we built a linear mixed model with the gaze feature, attention (on-task/unintentional MW/intentional MW), and their interaction as fixed effects (and picture type was entered as a covariate $)^{6}$. As shown in Table 3 , aggregated measures except for blinks consistently predicted memory performance across attentional states. Higher determinism was significantly associated with worse memory performance only during unintentional MW. CORM values were negatively associated with memory performance during both unintentional and intentional MW. Pairwise comparisons further showed that there was a stronger negative association between CORM and memory during intentional MW compared to when being on-task, $b=-.84, S E=.32, t=-2.60$, $p=0.03$. All other comparisons did not reveal significant differences between the simple slopes, $p s>.13$. Refer to the supplemental material for more results.

\section{Discussion}

The current study examined several sequential and aggregated measures of eye movements during MW in a scene perception task. We extended previous research by focusing on the sequential pattern (i.e., recurrence) of eye movements during both intentional and unintentional MW. MW is known to affect aggregated gaze measures, but impairments in the cognitive control of eye movement should also affect how looking plays out over time.

\section{Recurrence Patterns}

We found that scanpaths were more recurrent during unintentional MW than during on-task episodes. The increased recurrence during unintentional MW may be an adaptive response to perceptual decoupling. Superficial processing of the sensory input during the first run may force the viewer to start over and re-process those regions again. These results are in contrast with those of intentional MW, during which recurrence patterns were not significantly different from the on-task condition. During unintentional MW, participants might not be fully disengaged from the task goal, but instead may be drifting in and out of an on-task state. Re-fixations in this case may indicate specific attempts to re-engage attention to the task at hand. But if participants decided not

\footnotetext{
${ }^{6}$ Because recurrence rate, determinism, laminarity, CORM, and fixation duration were reported on a larger scale than memory performance (see Table 1), we divided the recurrence rate, determinism, laminarity, CORM by 100 and mean fixation duration by 1000 to avoid reporting very small numbers.
} 
a. On-task

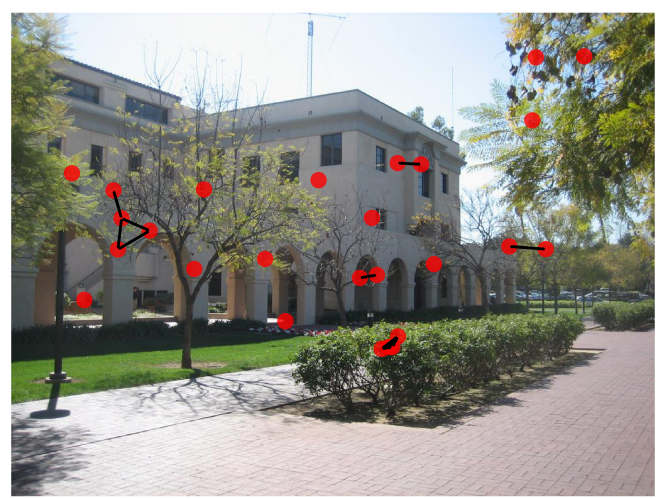

b. Unintentional MW

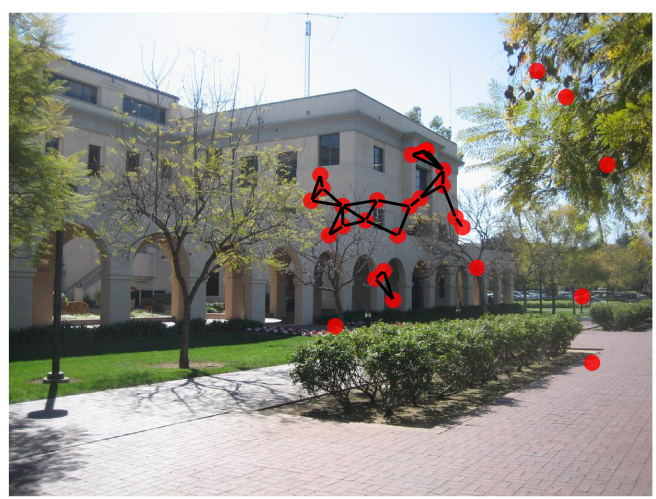

\section{Figure 4}

Representative scanpaths during unintentional $M W$ and on-task episodes. Each red point represents a single fixation. Recurrent fixations are connected by a black line. As shown in the figure, the scanpath during unintentional MW (on the right) was more repetitive compared to the on-task scanpath (on the left). A high-quality version of this figure can be found at https://osf.io/6pj9m/files/.

Table 3

The relationship between gaze features and memory of the picture under different attentional states.

\begin{tabular}{llll}
\hline & \multicolumn{1}{c}{ On-task } & \multicolumn{1}{c}{ MW: Unintentional } & \multicolumn{1}{c}{ MW: Intentional } \\
\hline Recurrence Rate & $-0.33[-0.62,-0.04] *$ & $-0.34[-0.71,0.03]$. & $-0.38[-0.97,0.21]$ \\
Determinism & $-0.08[-0.21,0.04]$ & $-0.21[-0.42,0.00] *$ & $0.01[-0.51,0.53]$ \\
Laminarity & $-0.07[-0.20,0.07]$ & $-0.06[-0.31,0.19]$ & $-0.04[-0.57,0.49]$ \\
CORM & $-0.12[-0.35,0.10]$ & $-0.41[-0.77,-0.05] *$ & $-0.96[-1.70,-0.22] * *$ \\
Fixation Dispersion & $0.47[0.20,0.74] * * *$ & $0.69[0.27,1.12] * * *$ & $1.20[0.36,2.03] * * *$ \\
Covered Area & $0.62[0.28,0.96] * * *$ & $1.05[0.48,1.62] * * *$ & $1.44[0.22,2.66] * *$ \\
Number of Fixations & $0.01[0.00,0.01] * * *$ & $0.01[0.00,0.02] * * *$ & $0.01[0.00,0.02] *$ \\
Mean Fixation Duration & $-0.33[-0.56,-0.10] * * *$ & $-0.50[-0.75,-0.25] * * *$ & $-0.75[-1.21,-0.28] * * *$ \\
Number of Blinks & $-0.01[-0.02,0.00]$ & $-0.01[-0.02,0.01]$ & $0.02[-0.02,0.05]$ \\
\hline
\end{tabular}

Note. Results are averaged over the levels of picture type. The brackets show $95 \%$ confidence intervals with Bonferroni correction. . $\mathrm{p}<.1 ; * \mathrm{p}<0.05 ; * * \mathrm{p}<0.01 ; * * * \mathrm{p}<0.001$.

to focus on the task, such recovery attempts seem unlikely even if visual processing fails. Interestingly, observers were also more likely to repeat entire scanpath segments during unintentional MW than when on task or intentionally MW (the level of determinism during intentional MW was indistinguishable from chance). Therefore, the adaptive response to perceptual decoupling may go beyond single re-fixations.

Of course, deficits in processing sensory input might not be the only reason that drives fixations back. As participants engage in task-unrelated thoughts, other components of the cognitive system such as visual working memory may also undergo serious disruptions. According to Zelinsky et al. (2011)'s “monitor-refixate-rehearse" model, an object's representation in visual working memory rapidly decays after gaze moves to new regions and a monitoring system can direct attention back to refresh its fading representation. Deficits in sensory processing and deficits in working memory are intricately related (Awh \& Jonides, 2001), and both can be seriously impacted during MW (e.g., Mrazek et al., 2012; Smallwood, Beach, et al., 2008). So it can be difficult to tease their effects apart. But overall, it appears that viewers were still able to detect processing deficits during unintentional MW and execute returning saccades to compensate for them.

One might reason that the increased recurrence and determinism during unintentional MW were caused by partici- 
pants repeatedly looking at the same location. Indeed, this viewing pattern would produce recurrence clusters on the recurrence plot that elevate both recurrence and determinism. However, this explanation is complicated by the lack of difference in laminarity and CORM between on-task and unintentional MW. Laminarity is a direct measure of repeatedly fixating the same region but there was little evidence that there was increased laminarity during unintentional MW. Moreover, repeatedly looking at the same location also implies that refixations occur soon after the initial fixation, which would lead to a smaller CORM value. Again, we did not find this to be the case. Therefore, it does seem that participants were revisiting different previous locations (i.e., specific scanpath sequences) instead of keeping eyes on the same location.

The current study found that repetitive scanpaths were associated with worse memory performance, which seems to speak against the idea that refixations are adaptive. Several factors could potentially affect memory besides re-fixations during encoding. First, having repetitive scanpaths may come at a cost of skipping other areas in the scene which may be also important for recognizing the picture. Second, it is not known how well the scanpaths during encoding and retrieval are coupled. The strongest version of the scanpath theory (Noton \& Stark, 1971b) predicts that reinstating the specific sequence of eye movements facilitate retrieval (for more discussions, see Foulsham \& Kingstone, 2013; Johansson et al., 2011; Richardson et al., 2009; Richardson \& Spivey, 2000). Unusual scanpath patterns (i.e., being more repetitive) during encoding may in fact be associated with worse coupling in scanpaths between encoding and retrieval, which may lead to worse recognition. Finally, the memory test assesses recognition of the entire picture rather than specific locations that were re-fixated. Recurrence at specific locations should be associated with better memory of those locations (e.g., Meghanathan et al., 2019; Tatler et al., 2005). In any case, repetitive looking alone does not seem to be sufficient to compensate for the deleterious effects of MW.

Another interesting finding is that the CORM value during intentional MW is a stronger predictor of memory performance compared to the CORM value when being on-task. In other words, if re-fixations tended to occur later during intentional MW, participants had worse memory of those pictures. This relationship was by itself significant during unintentional MW, but it did not significantly differ from the ontask condition. Overall, CORM was the highest during intentional MW compared to other attentional states, although the differences were not significant. One speculation is that, during intentional MW, participants deliberately disengaged from the task soon after the image was shown, and then came back to re-process the image when the presentation time was almost up. To the extent that participants engage in such be- havior during intentional MW, they remembered those pictures less well during the memory task.

\section{Aggregated Measures}

The increased recurrence during unintentional MW partially contributed to a decrease in the spatial spread of fixations (although a similar decrease in dispersion occurred during intentional MW as well). These findings were not consistent with Krasich et al. (2018), in which they observed a larger fixation dispersion during MW in their scene perception task. While both studies involve scene perception, there are some differences between the two tasks which might have resulted in this discrepancy. First, a part of the memory test in Krasich et al. (2018) required participants to identify small vignettes extracted from the full stimuli, which might have encouraged a skimming pattern during MW. Second, the stimuli in Krasich et al. (2018) appear to be more visually complex than the stimuli used in the current study. In particular, the scenes in Krasich et al. (2018) contained many objects that are rarely present in the current study, such as humans and Chinese characters. If during MW participants prefer to look at these objects (e.g., Zhang et al., 2020) and these objects happen to distribute widely across the scene, then fixation dispersion may increase. Third, the stimulus presentation time (45 - 75 sec) in Krasich et al. (2018) was much longer compared to the current study $(10 \mathrm{sec})$, and the increase in dispersion was only significant towards the end of presentation time. It is unknown whether we would have observed a similar pattern if we had extended the presentation time. In sum, those specific task parameters might modulate the relationship between eye movements and MW.

We found that MW was associated with fewer and longer fixations during scene perception, replicating findings in Krasich et al. (2018). It has been recently proposed that fixation duration reflects a stay-or-go evaluation of the benefit of staying at the current location versus the benefit of moving the eyes to a new location to gather new information (Tatler et al., 2017). During MW, visual processing may be less efficient or undergo more disruptions as attention decouples from the external stimuli. Therefore, the benefit of maintaining the current fixation may increase, which leads to prolonged fixation duration and fewer new fixations.

It is worth noting that fewer and longer fixations were not consistently associated with MW in many previous studies (e.g., Faber et al., 2020; Foulsham et al., 2013; Smilek et al., 2010; Steindorf \& Rummel, 2020; Uzzaman \& Joordens, 2011). While some of the discrepancies might reflect a power issue, the specific task setting may also modulate the relationship between eye movements and MW. For example, Faber et al. (2020) reported that MW was associated with fewer fixations during reading and scene perception, the same pattern did not emerge when watching a lec- 
ture or a film for the same group of participants. Thus, despite the similarity between these tasks at face value, gaze correlates of MW can be quite different. These findings may also challenge the notion that a task-general MW detector is possible by using exclusively global measures. The fact that global measures can be calculated independently of a specific task does not necessarily imply that these measures are not subject to the influence of the specific task (cf. Bixler \& D’Mello, 2016; Faber, Bixler, et al., 2018).

We also found that unintentional MW was associated with more blinks. A numerically similar pattern was observed for intentional MW. While the results are generally consistent with those in Smilek et al. (2010), the estimated differences ( $b=-0.26$ for unintentional MW and $b=-0.30$ for intentional MW) seem to be considerably smaller. Moreover, we found that unintentional MW was also associated with more extended periods of signal loss that are outside the typical range of blinking. These extended periods of signal loss may indicate micro-sleeps or a substantial change in head position, both of whom are likely to occur when task engagement is low (Caffier et al., 2003; Häkkänen et al., 1999; Schleicher et al., 2008; Seli et al., 2014). Therefore, by using gaze data alone it might be difficult to tease apart true blinks from other forms of data loss. Future research might benefit from combining eye-tracking with other validation methods (e.g., electroencephalogram and human coding of eye videos) when studying this question.

\section{Limitations and Future Directions}

To our knowledge, this is one of the first studies examining eye movement patterns during intentional MW. But unexpectedly, participants rarely reported intentional MW in our task. Real-world scenes usually present complex and meaningful information and thus participants may not want to deliberately disengage from the task (Faber, Radvansky, et al., 2018); intentional MW appears to occur more often in tasks with relatively simple and repetitive sensory input (Seli, Carriere, et al., 2018). Our preliminary results suggest that intentional and unintentional MW showed similar patterns for non-RQA measures. But differences may exist in how eye movements unfold over time. Because MW is a wideranging term, different sub-types of MW might not share the same set of gaze signatures. Analyzing scanpaths may help to unpack these potential differences in future research.

Another important future line of research would be to relate eye movements during MW to specific content in the scene. This question has been studied in the context of reading comprehension (e.g., the word frequency effect; Foulsham et al., 2013; Reichle et al., 2010; Steindorf \& Rummel, 2020). Recently, Henderson and Hayes (2017) showed that fixation placement across real-world scenes is driven by the meaningfulness of local regions. Therefore, it might be inter- esting to examine if eye movements are less associated with scene semantics during MW. Moreover, one study showed that, during MW, readers performed less re-reading from a joke's punchline, suggesting that they were less sensitive to semantic incongruity (Zhang et al., 2019). It would be interesting to examine if this finding can be extended to scene viewing. For example, during MW viewers may be less sensitive to semantically-incongruent information in the scene (e.g., a green stop sign).

There is an ongoing effort to develop gaze-based detectors of MW (e.g., Bixler \& D'Mello, 2016; Faber, Bixler, et al., 2018; Hutt et al., 2017). Given the fundamental role of scene perception in daily activities (e.g., driving), knowing when people are mind-wandering can have important implications for public welfare. Do scanpath measures bear any practical value in detecting MW? Scanpath measures are often more computationally complex than aggregated measures such as fixation count and dispersion, even though the two types of measures can be highly correlated (see Table 2). Therefore, the utility of scanpath measures may be a joint function of the computational burden they bring to the system and the unique predictive value beyond other more computationally efficient measures. However, this should not let us downplay the theoretical value scanpath measures can bring. Studying scanpaths provides a more nuanced understanding of how the online gaze control mechanism comes into play and how it might be affected during MW.

It is also worth noting that RQA only captures a single aspect (i.e., recurrence) of the highly complicated information existing in scanpaths, and there are many other analysis methods available. For example, the MultiMatch technique (Dewhurst et al., 2012) captures the similarity between two scanpaths in various dimensions, such as shape, direction, length, and duration. ScanMatch (Cristino et al., 2010), as another example, computes the overall similarity between scanpaths. These methods provide alternative ways to analyze eye movements during MW and on-task episodes. For example, scanpaths might be more similar to each other when attention is coupled to the task but become more idiosyncratic when individuals are mind-wandering. In sum, we believe that scanpath analysis, as a whole, provides a unique perspective on the current literature of MW and bears the potential to uncover more information about its underlying mechanisms.

\section{Conclusion}

To conclude, we found that scanpaths during unintentional MW were more repetitive compared to on-task episodes in a scene perception task. This increased repetitiveness may reflect an adaptive response to processing failures. On the other hand, we did not find a similar pattern during intentional MW, a period where participants deliberately disen- 
gaged from the task. In addition, we showed that both intentional and unintentional MW were associated with several aggregated gaze measures, including measures of the spatial spread of fixations, fixation count, fixation duration, and possibly eye-blinking. Understanding when, why, and in what attentional states observers repeat fixations will inform models of visual attention, and bring our understanding of attentional control much closer to real-life behaviors.

\section{References}

Altmann, G. T. (2004). Language-mediated eye movements in the absence of a visual world: The "blank screen paradigm". Cognition, 93(2), B79-B87.

Anderson, N. C., Bischof, W. F., Laidlaw, K. E. W., Risko, E. F., \& Kingstone, A. (2013). Recurrence quantification analysis of eye movements. Behavior Research Methods, 45(3), 842-856. https://doi.org/10.3758/ s13428-012-0299-5

Aust, F., \& Barth, M. (2020). papaja: Create APA manuscripts with R Markdown. https://github.com/ $\mathrm{crsh} /$ papaja

Awh, E., \& Jonides, J. (2001). Overlapping mechanisms of attention and spatial working memory. Trends in Cognitive Sciences, 5(3), 119-126.

Barr, D. J., Levy, R., Scheepers, C., \& Tily, H. J. (2013). Random effects structure for confirmatory hypothesis testing: Keep it maximal. Journal of Memory and Language. https://doi.org/10.1016/j.jml.2012. 11.001

Barron, E., Riby, L. M., Greer, J., \& Smallwood, J. (2011). Absorbed in thought: The effect of mind wandering on the processing of relevant and irrelevant events. Psychological Science, 22(5), 596-601. https://doi. org/10.1177/0956797611404083

Bates, D., Mächler, M., Bolker, B., \& Walker, S. (2015). Fitting linear mixed-effects models using lme4. Journal of Statistical Software, 67(1), 1-48. https://doi. org/10.18637/jss.v067.i01

Bixler, R., \& D’Mello, S. (2016). Automatic gaze-based user-independent detection of mind wandering during computerized reading. User Modeling and User-Adapted Interaction, 26(1), 33-68. https:// doi.org/10.1007/s11257-015-9167-1

Bochynska, A., \& Laeng, B. (2015). Tracking down the path of memory: Eye scanpaths facilitate retrieval of visuospatial information. Cognitive Processing, 16(1), 159-163.

Braboszcz, C., \& Delorme, A. (2011). Lost in thoughts:
Neural markers of low alertness during mind wandering. NeuroImage, 54(4), 3040-3047. https: //doi.org/10.1016/j.neuroimage.2010.10.008

Caffier, P. P., Erdmann, U., \& Ullsperger, P. (2003). Experimental evaluation of eye-blink parameters as a drowsiness measure. European Journal of Applied Physiology, 89(3), 319-325. https://doi.org/ 10.1007/s00421-003-0807-5

Castelhano, M. S., \& Rayner, K. (2008). Eye movements during reading, visual search, and scene perception: An overview. In Cognitive and cultural influences on eye movements (Vol. 2175, pp. 3-33).

Christoff, K., Mills, C., Andrews-Hanna, J. R., Irving, Z. C., Thompson, E., Fox, K. C. R., \& Kam, J. W. Y. (2018). Mind-wandering as a scientific concept: Cutting through the definitional haze. Trends in Cognitive Sciences, 22(11), 957-959. https://doi. org/10.1016/j.tics.2018.07.004

Conigrave, J. (2020). Corx: Create and format correlation matrices. https://CRAN.R-project.org/package= corx

Cristino, F., Mathôt, S., Theeuwes, J., \& Gilchrist, I. D. (2010). ScanMatch: A novel method for comparing fixation sequences. Behavior Research Methods, 42(3), 692-700. https://doi.org/10.3758/BRM. 42.3.692

Dalmaijer, E. S., Mathôt, S., \& Stigchel, S. der. (2014). PyGaze: An open-source, cross-platform toolbox for minimal-effort programming of eyetracking experiments. Behavior Research Methods, 46(4), 913-921.

Dewhurst, R., Foulsham, T., Jarodzka, H., Johansson, R., Holmqvist, K., \& Nyström, M. (2018). How task demands influence scanpath similarity in a sequential number-search task. Vision Research, 149, 923. https://doi.org/10.1016/j.visres.2018.05.006

Dewhurst, R., Nyström, M., Jarodzka, H., Foulsham, T., Johansson, R., \& Holmqvist, K. (2012). It depends on how you look at it: Scanpath comparison in multiple dimensions with MultiMatch, a vector-based approach. Behavior Research Methods, 44(4), 10791100 .

DeWitt, P. (2019). Qwraps2: Quick wraps 2. https://CRAN. $\mathrm{R}$-project.org/package $=$ qwraps 2

Droll, J. A., \& Hayhoe, M. M. (2007). Trade-offs between gaze and working memory use. Journal of Experimental Psychology: Human Perception and Performance, 33(6), 1352-1365. https://doi.org/10.1037/ 
0096-1523.33.6.1352

Ehrlichman, H., Micic, D., Sousa, A., \& Zhu, J. (2007). Looking for answers: Eye movements in non-visual cognitive tasks. Brain and Cognition, 64(1), 7-20. https://doi.org/10.1016/j.bandc.2006.10.001

Faber, M., Bixler, R., \& D’Mello, S. K. (2018). An automated behavioral measure of mind wandering during computerized reading. Behavior Research Methods, 50(1), 134-150. https://doi.org/10.3758/ s13428-017-0857-y

Faber, M., Krasich, K., Bixler, R., Brockmole, J., \& D'Mello, S. (2020). The eye-mind wandering link: Identifying gaze indices of mind wandering across tasks. Journal of Experimental Psychology: Human Perception and Performance.

Faber, M., Radvansky, G. A., \& D’Mello, S. K. (2018). Driven to distraction: A lack of change gives rise to mind wandering. Cognition, 173, 133-137. https: //doi.org/10.1016/j.cognition.2018.01.007

Foulsham, T., Farley, J., \& Kingstone, A. (2013). Mind wandering in sentence reading: Decoupling the link between mind and eye. Canadian Journal of Experimental Psychology/Revue Canadienne de Psychologie Expérimentale, 67(1), 51.

Foulsham, T., \& Kingstone, A. (2013). Fixation-dependent memory for natural scenes: An experimental test of scanpath theory. Journal of Experimental Psychology: General, 142(1), 41-56. https://doi.org/ $10.1037 / \mathrm{a} 0028227$

Foulsham, T., \& Underwood, G. (2008). What can saliency models predict about eye movements? Spatial and sequential aspects of fixations during encoding and recognition. Journal of Vision, 8(2), 6. https://doi. org/10.1167/8.2.6

Fox, K. C. R., Spreng, R. N., Ellamil, M., Andrews-Hanna, J. R., \& Christoff, K. (2015). The wandering brain: Meta-analysis of functional neuroimaging studies of mind-wandering and related spontaneous thought processes. NeuroImage, 111, 611-621. https://doi. org/10.1016/j.neuroimage.2015.02.039

Frank, D. J., Nara, B., Zavagnin, M., Touron, D. R., \& Kane, M. J. (2015). Validating older adults' reports of less mind-wandering: An examination of eye movements and dispositional influences. Psychology and Aging, 30(2), 266-278. https://doi.org/ 10.1037/pag0000031

Gilchrist, I. D., \& Harvey, M. (2000). Refixation frequency and memory mechanisms in visual search. Cur- rent Biology, 10(19), 1209-1212. https://doi.org/ 10.1016/S0960-9822(00)00729-6

Golchert, J., Smallwood, J., Jefferies, E., Seli, P., Huntenburg, J. M., Liem, F., Lauckner, M. E., Oligschläger, S., Bernhardt, B. C., Villringer, A., \& Margulies, D. S. (2017). Individual variation in intentionality in the mind-wandering state is reflected in the integration of the default-mode, fronto-parietal, and limbic networks. NeuroImage, 146, 226-235. https: //doi.org/10.1016/j.neuroimage.2016.11.025

Grandchamp, R., Braboszcz, C., \& Delorme, A. (2014). Oculometric variations during mind wandering. Frontiers in Psychology, 5. https://doi.org/10.3389/ fpsyg.2014.00031

Gurtner, L. M., Bischof, W. F., \& Mast, F. W. (2019). Recurrence quantification analysis of eye movements during mental imagery. Journal of Vision, 19(1), 17. https://doi.org/10.1167/19.1.17

Häkkänen, H., LicPsych, Summala, H., Partinen, M., Tiihonen, M., \& Silvo, J. (1999). Blink duration as an indicator of driver sleepiness in professional bus drivers. Sleep, 22(6), 798-802. https://doi.org/10. 1093/sleep/22.6.798

Henderson, J. M. (2003). Human gaze control during realworld scene perception. Trends in Cognitive Sciences, 7(11), 498-504.

Henderson, J. M., \& Hayes, T. R. (2017). Meaning-based guidance of attention in scenes as revealed by meaning maps. Nature Human Behaviour, 1, 7.

Hershman, R., Henik, A., \& Cohen, N. (2018). A novel blink detection method based on pupillometry noise. $\mathrm{Be}$ havior Research Methods, 50(1), 107-114. https: //doi.org/10.3758/s13428-017-1008-1

Hutt, S., Mills, C., Bosch, N., Krasich, K., Brockmole, J., \& D'Mello, S. (2017). "Out of the fr-eye-ing pan": Towards gaze-based models of attention during learning with technology in the classroom. Proceedings of the 25th Conference on User Modeling, Adaptation and Personalization, 94-103. https: //doi.org/10.1145/3079628.3079669

Johansson, R., Holsanova, J., Dewhurst, R., \& Holmqvist, K. (2011). Eye movements during pictorial recall have a functional role, but they are not reinstatements of those from encoding. Journal of Eye Movement Research, 4(3), 32-32.

Johansson, R., \& Johansson, M. (2014). Look here, eye movements play a functional role in memory retrieval. Psychological Science, 25(1), 236-242. 
Jubera-García, E., Gevers, W., \& Van Opstal, F. (2019). Influence of content and intensity of thought on behavioral and pupil changes during active mindwandering, off-focus, and on-task states. Attention, Perception, \& Psychophysics. https://doi.org/ 10.3758/s13414-019-01865-7

Kam, J. W. Y., Dao, E., Farley, J., Fitzpatrick, K., Smallwood, J., Schooler, J. W., \& Handy, T. C. (2011). Slow fluctuations in attentional control of sensory cortex. Journal of Cognitive Neuroscience, 23(2), 460-470. https://doi.org/10.1162/jocn.2010.21443

Kane, M. J., Brown, L. H., McVay, J. C., Silvia, P. J., MyinGermeys, I., \& Kwapil, T. R. (2007). For whom the mind wanders, and when: An experience-sampling study of working memory and executive control in daily life. Psychological Science, 18(7), 614-621.

Kane, M. J., Gross, G. M., Chun, C. A., Smeekens, B. A., Meier, M. E., Silvia, P. J., \& Kwapil, T. R. (2017). For whom the mind wanders, and when, varies across laboratory and daily-life settings. Psychological Science, 28(9), 1271-1289. https://doi. org/10.1177/0956797617706086

Kane, M. J., Smeekens, B. A., Bastian, C. C. von, Lurquin, J. H., Carruth, N. P., \& Miyake, A. (2017). A combined experimental and individual-differences investigation into mind wandering during a video lecture. Journal of Experimental Psychology: General, 146(11), 1649-1674. https://doi.org/10.1037/ xge0000362

Körner, C., \& Gilchrist, I. D. (2008). Memory processes in multiple-target visual search. Psychological Research, 72(1), 99-105.

Krasich, K., McManus, R., Hutt, S., Faber, M., D’Mello, S. K., \& Brockmole, J. R. (2018). Gaze-based signatures of mind wandering during real-world scene processing. Journal of Experimental Psychology: General, 147(8), 1111-1124. https://doi.org/10. 1037/xge0000411

Kuznetsova, A., Brockhoff, P. B., \& Christensen, R. H. B. (2017). lmerTest package: Tests in linear mixed effects models. Journal of Statistical Software, 82(13), 1-26. https://doi.org/10.18637/jss.v082.i13

Land, M. F., \& Hayhoe, M. (2001). In what ways do eye movements contribute to everyday activities? $\mathrm{Vi}$ sion Research, 41(25), 3559-3565.

Lenth, R. (2019). Emmeans: Estimated marginal means, aka least-squares means. https://CRAN.R-project.org/ package $=$ emmeans
Lindquist, S. I., \& McLean, J. P. (2011). Daydreaming and its correlates in an educational environment. Learning and Individual Differences, 21(2), 158167. https://doi.org/10.1016/j.lindif.2010.12.006

Luke, S. G., \& Henderson, J. M. (2013). Oculomotor and cognitive control of eye movements in reading: Evidence from mindless reading. Attention, Perception, \& Psychophysics, 75(6), 1230-1242.

Malsburg, T. von der, Kliegl, R., \& Vasishth, S. (2015). Determinants of scanpath regularity in reading. Cognitive Science, 39(7), 1675-1703. https://doi.org/10. 1111/cogs. 12208

Malsburg, T. von der, \& Vasishth, S. (2011). What is the scanpath signature of syntactic reanalysis? Journal of Memory and Language, 65(2), 109-127. https: //doi.org/10.1016/j.jml.2011.02.004

Malsburg, T. von der, \& Vasishth, S. (2013). Scanpaths reveal syntactic underspecification and reanalysis strategies. Language and Cognitive Processes, 28(10), 1545-1578. https://doi.org/10.1080/ 01690965.2012 .728232

Mathôt, S., Schreij, D., \& Theeuwes, J. (2012). OpenSesame: An open-source, graphical experiment builder for the social sciences. Behavior Research Methods, 44(2), 314-324.

McIntyre, N. A., \& Foulsham, T. (2018). Scanpath analysis of expertise and culture in teacher gaze in realworld classrooms. Instructional Science, 46(3), 435-455.

Meghanathan, R. N., Nikolaev, A. R., \& Leeuwen, C. van. (2019). Refixation patterns reveal memoryencoding strategies in free viewing. Attention, Perception, \& Psychophysics, 81(7), 2499-2516. https: //doi.org/10.3758/s13414-019-01735-2

Mooneyham, B. W., \& Schooler, J. W. (2013). The costs and benefits of mind-wandering: A review. Canadian Journal of Experimental Psychology/Revue Canadienne de Psychologie Expérimentale, 67(1), 1118. https://doi.org/10.1037/a0031569

Mrazek, M. D., Smallwood, J., Franklin, M. S., Chin, J. M., Baird, B., \& Schooler, J. W. (2012). The role of mind-wandering in measurements of general aptitude. Journal of Experimental Psychology: General, 141(4), 788.

Noton, D., \& Stark, L. (1971a). Eye movements and visual perception. Scientific American, 224(6), 34-43.

Noton, D., \& Stark, L. (1971b). Scanpaths in eye movements during pattern perception. Science, 171(3968), 
$308-311$.

Oliva, A., \& Torralba, A. (2006). Building the gist of a scene: The role of global image features in recognition. Progress in Brain Research, 155, 23-36.

Phillips, N. E., Mills, C., D’Mello, S., \& Risko, E. F. (2016). On the influence of re-reading on mind wandering. Quarterly Journal of Experimental Psychology, 69(12), 2338-2357. https://doi.org/10.1080/ 17470218.2015.1107109

Rayner, K., \& Fischer, M. H. (1996). Mindless reading revisited: Eye movements during reading and scanning are different. Perception \& Psychophysics, 58(5), 734-747.

Rayner, K., Smith, T. J., Malcolm, G. L., \& Henderson, J. M. (2009). Eye movements and visual encoding during scene perception. Psychological Science, 20(1), 6-10. https://doi.org/10.1111/j.14679280.2008.02243.x

R Core Team. (2019). R: A language and environment for statistical computing. R Foundation for Statistical Computing. https://www.R-project.org/

Reichle, E. D., Reineberg, A. E., \& Schooler, J. W. (2010). Eye movements during mindless reading. Psychological Science, 21(9), 1300-1310.

Richardson, D. C., Altmann, G. T., Spivey, M. J., \& Hoover, M. A. (2009). Much ado about eye movements to nothing: A response to ferreira et al.: taking a new look at looking at nothing. Trends in Cognitive Sciences, 13(6), 235-236.

Richardson, D. C., \& Spivey, M. J. (2000). Representation, space and hollywood squares: Looking at things that aren't there anymore. Cognition, 76(3), 269295.

Robison, M. K., Miller, A. L., \& Unsworth, N. (2019). Examining the effects of probe frequency, response options, and framing within the thought-probe method. Behavior Research Methods, 51(1), 398408. https://doi.org/10.3758/s13428-019-01212-6

Russell, B. C., Torralba, A., Murphy, K. P., \& Freeman, W. T. (2008). LabelMe: A database and web-based tool for image annotation. International Journal of Computer Vision, 77(1), 157-173.

Schleicher, R., Galley, N., Briest, S., \& Galley, L. (2008). Blinks and saccades as indicators of fatigue in sleepiness warnings: Looking tired? $\mathrm{Er}$ gonomics, 51(7), 982-1010. https://doi.org/10. 1080/00140130701817062
Schooler, J. W., Smallwood, J., Christoff, K., Handy, T. C., Reichle, E. D., \& Sayette, M. A. (2011). Metaawareness, perceptual decoupling and the wandering mind. Trends in Cognitive Sciences, 15(7), 319326. https://doi.org/10.1016/j.tics.2011.05.006

Schotter, E. R., Tran, R., \& Rayner, K. (2014). Don't believe what you read (only once): Comprehension is supported by regressions during reading. Psychological Science. https://doi.org/10.1177/ 0956797614531148

Seli, P., Beaty, R. E., Cheyne, J. A., Smilek, D., Oakman, J., \& Schacter, D. L. (2018). How pervasive is mind wandering, really?, Consciousness and Cognition, 66, 74-78. https://doi.org/10.1016/j.concog.2018. 10.002

Seli, P., Carriere, J. S. A., Levene, M., \& Smilek, D. (2013). How few and far between? Examining the effects of probe rate on self-reported mind wandering. Frontiers in Psychology, 4. https://doi.org/10.3389/ fpsyg.2013.00430

Seli, P., Carriere, J. S. A., Thomson, D. R., Cheyne, J. A., Martens, K. A. E., \& Smilek, D. (2014). Restless mind, restless body. Journal of Experimental Psychology: Learning, Memory, and Cognition, 40(3), 660-668. https://doi.org/10.1037/a0035260

Seli, P., Carriere, J. S. A., Wammes, J. D., Risko, E. F., Schacter, D. L., \& Smilek, D. (2018). On the clock: Evidence for the rapid and strategic modulation of mind wandering. Psychological Science, 29(8), 1247-1256. https://doi.org/10.1177/ 0956797618761039

Seli, P., Cheyne, J. A., Xu, M., Purdon, C., \& Smilek, D. (2015). Motivation, intentionality, and mind wandering: Implications for assessments of taskunrelated thought. Journal of Experimental Psychology: Learning, Memory, and Cognition, 41(5), 1417.

Seli, P., Kane, M. J., Metzinger, T., Smallwood, J., Schacter, D. L., Maillet, D., Schooler, J. W., \& Smilek, D. (2018). The family-resemblances framework for mind-wandering remains well clad. Trends in Cognitive Sciences, 22(11), 959-961. https://doi.org/ 10.1016/j.tics.2018.07.007

Seli, P., Kane, M. J., Smallwood, J., Schacter, D. L., Maillet, D., Schooler, J. W., \& Smilek, D. (2018). Mind-wandering as a natural kind: A familyresemblances view. Trends in Cognitive Sciences, 22(6), 479-490. https://doi.org/10.1016/j.tics.2018. 03.010 
Seli, P., Risko, E. F., \& Smilek, D. (2016). On the necessity of distinguishing between unintentional and intentional mind wandering. Psychological Science, 27(5), 685-691. https://doi.org/10.1177/ 0956797616634068

Seli, P., Risko, E. F., Smilek, D., \& Schacter, D. L. (2016). Mind-wandering with and without intention. Trends in Cognitive Sciences, 20(8), 605-617. https://doi. org/10.1016/j.tics.2016.05.010

Seli, P., Schacter, D. L., Risko, E. F., \& Smilek, D. (2017). Increasing participant motivation reduces rates of intentional and unintentional mind wandering. Psychological Research. https://doi.org/10. 1007/s00426-017-0914-2

Seli, P., Smallwood, J., Cheyne, J. A., \& Smilek, D. (2015). On the relation of mind wandering and ADHD symptomatology. Psychonomic Bulletin \& Review, 22(3), 629-636. https://doi.org/10.3758/s13423014-0793-0

Singmann, H., Bolker, B., Westfall, J., Aust, F., \& BenShachar, M. S. (2019). Afex: Analysis of factorial experiments. https:/CRAN.R-project.org/ package $=$ afex

Smallwood, J. (2011). Mind-wandering while reading: Attentional decoupling, mindless reading and the cascade model of inattention: Mind-wandering while reading. Language and Linguistics Compass, 5(2), 63-77. https://doi.org/10.1111/j.1749-818X.2010. 00263.x

Smallwood, J. (2013). Distinguishing how from why the mind wanders: A process-occurrence framework for self-generated mental activity. Psychological Bulletin, 139(3), 519-535. https://doi.org/10.1037/ a0030010

Smallwood, J., Beach, E., Schooler, J. W., \& Handy, T. C. (2008). Going AWOL in the brain: Mind wandering reduces cortical analysis of external events. Journal of Cognitive Neuroscience, 20(3), 458-469.

Smallwood, J., McSpadden, M., \& Schooler, J. W. (2008). When attention matters: The curious incident of the wandering mind. Memory \& Cognition, 36(6), 1144-1150. https://doi.org/10.3758/MC.36.6.1144

Smilek, D., Carriere, J. S. A., \& Cheyne, J. A. (2010). Out of mind, out of sight: Eye blinking as indicator and embodiment of mind wandering. Psychological Science, 21(6), 786-789. http://www.jstor.org/ stable/41062288

Spivey, M. J., \& Geng, J. J. (2001). Oculomotor mechanisms activated by imagery and memory: Eye movements to absent objects. Psychological Research, 65(4), 235-241.

Steindorf, L., \& Rummel, J. (2020). Do your eyes give you away? A validation study of eye-movement measures used as indicators for mindless reading. $\mathrm{Be}$ havior Research Methods, 52(1), 162-176.

Tatler, B. W., Brockmole, J. R., \& Carpenter, R. H. S. (2017). LATEST: A model of saccadic decisions in space and time. Psychological Review, 124(3), 267-300. https://doi.org/10.1037/rev0000054

Tatler, B. W., Gilchrist, I. D., \& Land, M. F. (2005). Visual memory for objects in natural scenes: From fixations to object files. The Quarterly Journal of Experimental Psychology Section A, 58(5), 931-960. https://doi.org/10.1080/02724980443000430

Tatler, B. W., Hayhoe, M. M., Land, M. F., \& Ballard, D. H. (2011). Eye guidance in natural vision: Reinterpreting salience. Journal of Vision, 11(5), 5-5.

Torralba, A., Oliva, A., Castelhano, M. S., \& Henderson, J. M. (2006). Contextual guidance of eye movements and attention in real-world scenes: The role of global features in object search. Psychological Review, 113(4), 766.

Tremblay, S., Saint-Aubin, J., \& Jalbert, A. (2006). Rehearsal in serial memory for visual-spatial information: Evidence from eye movements. Psychonomic Bulletin \& Review, 13(3), 452-457. https: //doi.org/10.3758/BF03193869

Unsworth, N., \& Robison, M. K. (2016). Pupillary correlates of lapses of sustained attention. Cognitive, Affective, \& Behavioral Neuroscience, 16(4), 601-615. https://doi.org/10.3758/s13415-016-0417-4

Uzzaman, S., \& Joordens, S. (2011). The eyes know what you are thinking: Eye movements as an objective measure of mind wandering. Consciousness and Cognition, 20(4), 1882-1886. https://doi.org/10. 1016/j.concog.2011.09.010

Wang, Y., Toor, S. S., Gautam, R., \& Henson, D. B. (2011). Blink frequency and duration during perimetry and their relationship to test-retest threshold variability. Investigative Ophthalmology \& Visual Science, 52(7), 4546-4550. https://doi.org/10.1167/iovs.106553

Wickham, H., Averick, M., Bryan, J., Chang, W., McGowan, L. D., François, R., Grolemund, G., Hayes, A., Henry, L., Hester, J., Kuhn, M., Pedersen, T. L., Miller, E., Bache, S. M., Müller, K., Ooms, J., 
Robinson, D., Seidel, D. P., Spinu, V., ... Yutani, H. (2019). Welcome to the tidyverse. Journal of Open Source Software, 4(43), 1686. https: //doi.org/10.21105/joss.01686

Xiao, J., Hays, J., Ehinger, K. A., Oliva, A., \& Torralba, A. (2010). Sun database: Large-scale scene recognition from abbey to zoo. 2010 IEEE Computer Society Conference on Computer Vision and Pattern Recognition, 3485-3492.

Xie, Y. (2015). Dynamic documents with $R$ and knitr (2nd ed.). Chapman; Hall/CRC. https://yihui.org/knitr/

Yanko, M. R., \& Spalek, T. M. (2014). Driving with the wandering mind: The effect that mind-wandering has on driving performance. Human Factors: The Journal of the Human Factors and Ergonomics Society, 56(2), 260-269. https://doi.org/10.1177/ 0018720813495280

Yarbus, A. L. (1967). Eye movements during perception of complex objects. In Eye movements and vision (pp. 171-211). Springer US. https://doi.org/10. 1007/978-1-4899-5379-7_8

Zelinsky, G. J., Loschky, L. C., \& Dickinson, C. A. (2011). Do object refixations during scene viewing indicate rehearsal in visual working memory? Memory \& Cognition, 39(4), 600-613. https://doi.org/10.3758/ s13421-010-0048-x

Zhang, H., Miller, K. F., Sun, X., \& Cortina, K. S. (2020). Wandering eyes: Eye movements during mind wandering in video lectures. Applied Cognitive Psychology, 34(2), acp.3632. https://doi.org/10.1002/ acp. 3632

Zhang, H., Qu, C., Miller, K. F., \& Cortina, K. S. (2019). Missing the joke: Reduced rereading of garden-path jokes during mind-wandering. Journal of Experimental Psychology: Learning, Memory, and Cognition. https://doi.org/10.1037/xlm0000745

Zhu, H. (2019). KableExtra: Construct complex table with 'kable' and pipe syntax. https://CRAN.R-project. org/package=kableExtra 


\section{Appendix A \\ Mini-review of Published Studies Examining Gaze Patterns of Mind-wandering}

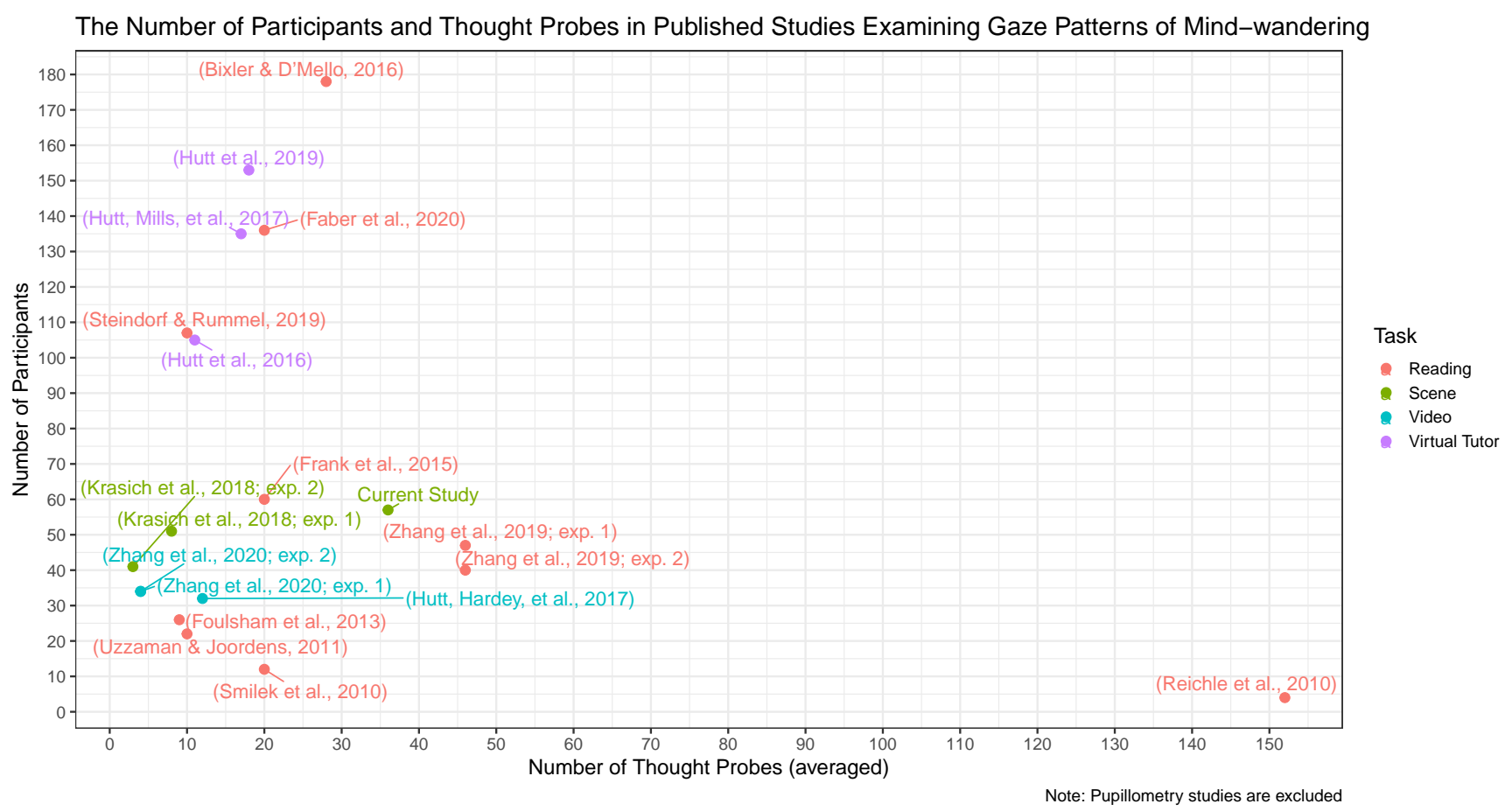

\section{Figure A1}

The Number of Participants and Thought Probes in Published Studies Examining Gaze Patterns of Mind-wandering 


\section{Appendix B \\ Validation of RQA Measures}

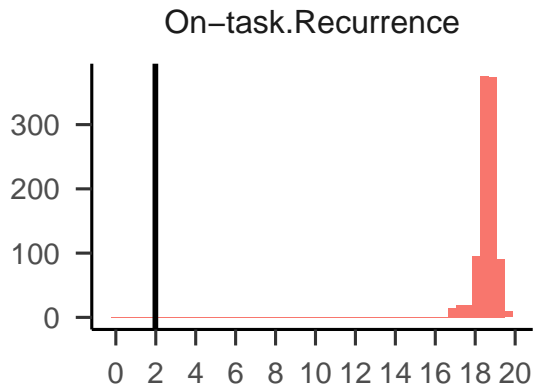

MW: Unintentional.Recurrence

MW: Intentional.Recurrence
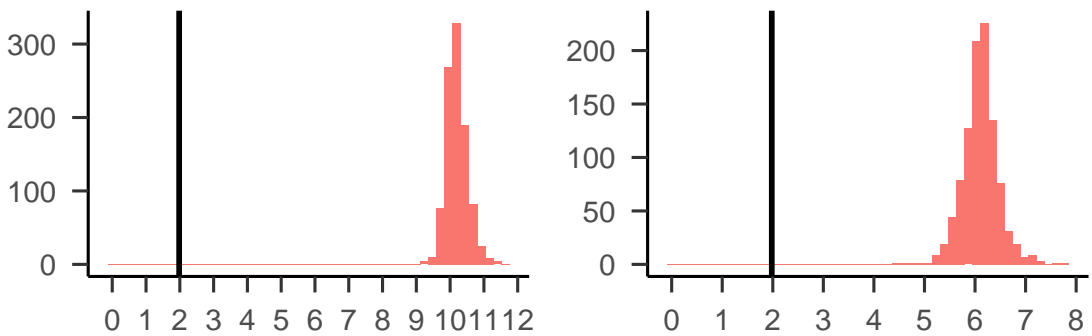

VW: Unintentional.Determinism

MW: Intentional.Determinism
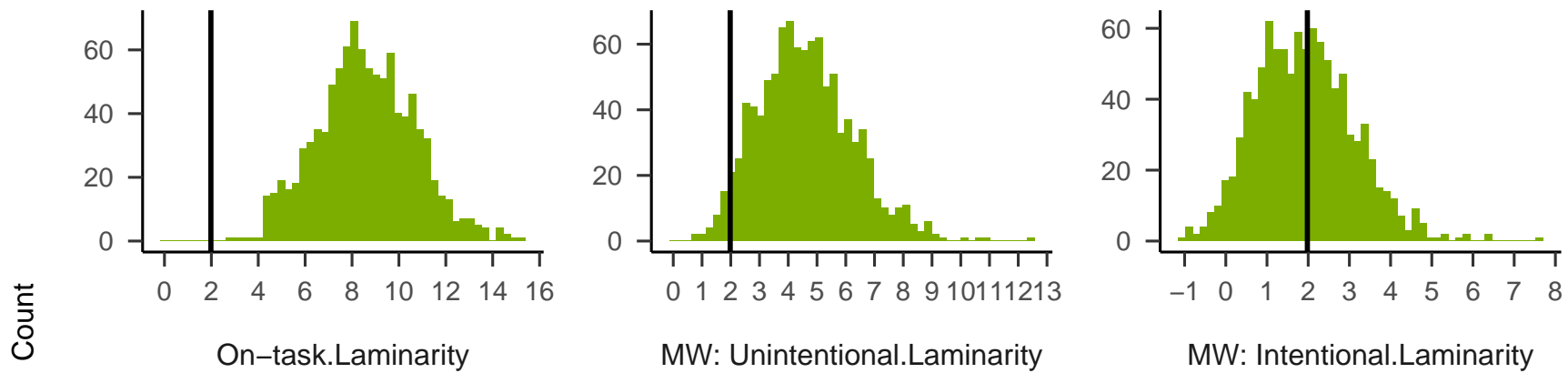

MW: Unintentional.Laminarity
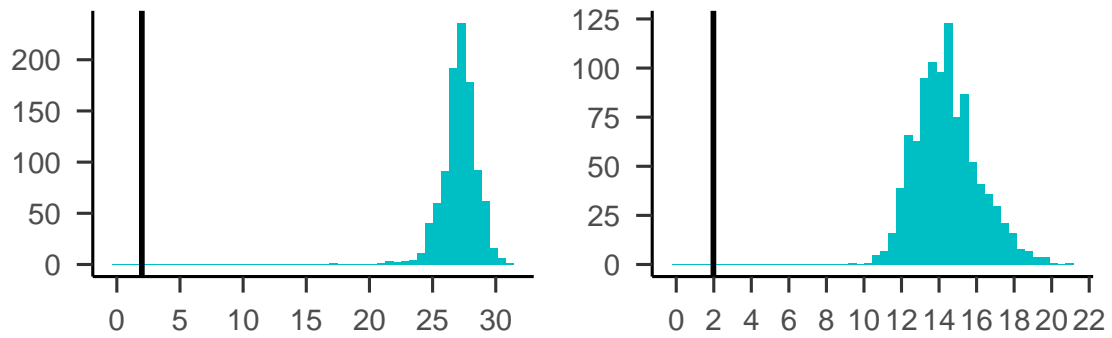

MW: Intentional.Laminarity

On-task.CORM

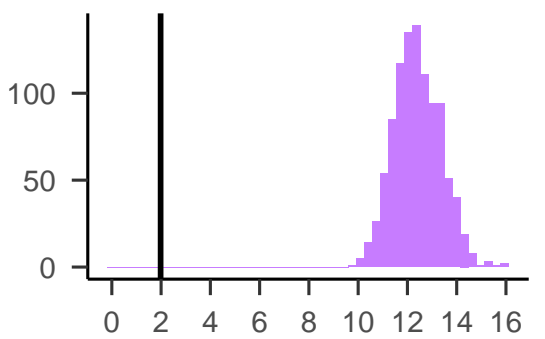

MW: Unintentional.CORM
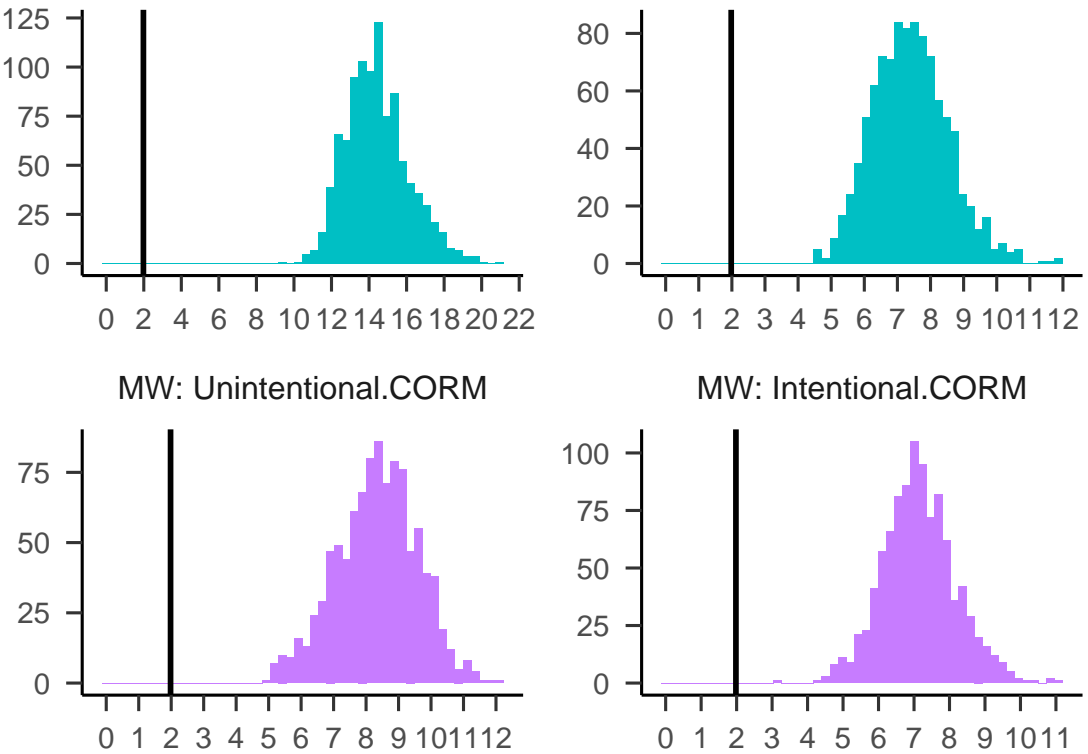

MW: Intentional.CORM

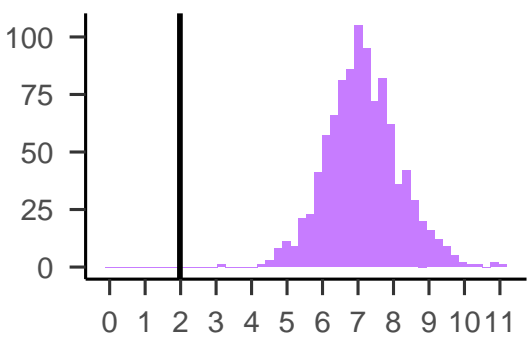

t-values

\section{Figure B1}

Distributions of t-values from 1000 one-sample t-tests examining the difference between empirical values and values derived from random fixation sequences against 0 . The vertical black line indicates the critical $* * v a l u e(1.67$ when $N=57$, one-tailed). All empirical values were above-chance except for determinism during intentional MW. 